International Journal of Pure and Applied Mathematics

Volume 95 No. 2 2014, 253-296

ISSN: 1311-8080 (printed version); ISSN: 1314-3395 (on-line version)

url: http://www.ijpam.eu

doi: http://dx.doi.org/10.12732/ijpam.v95i2.12

ijpam.eu

\title{
EXISTENCE AND UNIQUENESS OF SOLUTIONS FOR DAMPED LINEAR HYPERBOLIC EQUATIONS WITH DIRICHLET BOUNDARY CONDITIONS
}

\author{
Hacene Bennour ${ }^{1} \S$, Mohamed Said Said ${ }^{2}$ \\ ${ }^{1}$ Department of Mathematics \\ Kasdi Merbah- Ouargla University \\ Ouargla, 30000, ALGERIA \\ ${ }^{2}$ Department of Mathematics \\ Kasdi Merbah- Ouargla University \\ Ouargla, 30000, ALGERIA
}

\begin{abstract}
We consider damped linear hyperbolic equations with Dirichlet boundary conditions. We prove the existence, uniqueness, and regularity of the solution. We apply semi-discretization in time technique.
\end{abstract}

AMS Subject Classification: 35A01, 35A02, 35L20

Key Words: linear hyperbolic equations, damping, Faedo-Galerkin method, discretization in time

\section{Introduction}

In this paper, we study the initial boundary value problem for the following damped linear hyperbolic equations with Dirichlet boundary conditions.

$$
\left\{\begin{array}{r}
\left.\frac{\partial^{2} u}{\partial t^{2}}+\eta \frac{\partial u}{\partial t}-\Delta u=f(x, t), x \in \Omega, t \in\right] 0, T[, \\
u(t, x)=0, \text { on } \Sigma, \\
u(0, x)=u_{0}(x), \frac{\partial u(0, x)}{\partial t}=u_{1}(x), x \in \Omega .
\end{array}\right.
$$

where $\eta$ is the constant damping coefficient, $\eta \in \mathbb{R}$ and $T$ finite, $\Omega$ is a bounded open domain in $\mathbb{R}^{n}, n \geq 1$, with a smooth boundary $\partial \Omega$. We denote by $Q$ the

Received: May 7, 2014

(c) 2014 Academic Publications, Ltd.

$\S$ Correspondence author url: www.acadpubl.eu 
cylinder of $\left.\mathbb{R}_{x}^{n} \times \mathbb{R}_{t}, Q=\Omega \times\right] 0, T[$ and by $\Sigma$ the lateral boundary of $Q, \Sigma=$ $\partial \Omega \times] 0, T\left[, \Delta u=\Sigma_{i=1}^{n} \frac{\partial^{2} u}{\partial x_{i}^{2}}\right.$ is the Laplacian, and linear function $\left.f: \Omega \times\right] 0, T[\longrightarrow$ $\mathbb{R}$ for $i=0,1$, the functions $u_{i}: \Omega \longrightarrow \mathbb{R}$, are given. we find a function $u=u(x, t)$, is a real-valued satisfies $(1)$.

This Problem has its origin in a physical problem, we study a model that describes the transverse vibrations of a membrane $\Omega$ fixed at its ends and in the presence of damping $\eta$. Let $u(x, t)$ be the vertical position of $x \in \Omega$ at time $t \in[0, T]$, is retarded by a damping force proportional to the velocity of the membrane, then $u$ satisfies (1).

This problem has been already investigated by many authors.

For example in $([2]),([7]),([17])$.

We define some function spaces required to establish the existence and uniqueness of solution to (1). We use the function spaces for any $1 \leq p<\infty$, $L^{p}(\Omega)$ is the space of real measurable functions $u: \Omega \longrightarrow \mathbb{R}$ for the Lebesgue measure $d x$, it is a Banach space for the following norm

$$
\|u\|_{L^{p}(\Omega)}=\left(\int_{\Omega}|u(x)|^{p} d x\right)^{\frac{1}{p}}<+\infty
$$

for $p=2, L^{2}(\Omega)$ is a Hilbert space for the scalar product

$$
(u, v)=\int_{\Omega} u(x) v(x) d x,
$$

the corresponding norm being denoted $\|u\|$,

$$
\|u\|=\left(\int_{\Omega} u^{2}(x) d x\right)^{\frac{1}{2}} .
$$

if $X$ is a Banach space, $1 \leq p<\infty, L^{p}(0, T ; X)$ is the space of measurable functions $u$ of $] 0, T$ [ into $X$ for the Lebesgue measure $d t$, which is Banach space for the following norm

$$
\|u\|_{L^{p}(0, T ; X)}=\left(\int_{0}^{T}\|u(t)\|_{X}^{p} d t\right)^{\frac{1}{p}}<+\infty
$$

if $X=L^{p}(\Omega)$, then $L^{p}(] 0, T\left[; L^{p}(\Omega)\right)=L^{p}(Q)$.

$L^{\infty}(0, T ; X)$ is the space of measurable functions from $] 0, T[$ into $X$ which are essentially bounded, the space is Banach for the following norm

$$
\|u\|_{L^{\infty}(0, T ; X)}=\sup _{t \in] 0, T[} \operatorname{ess}\|u(t)\|_{X}
$$


we denote by $\mathscr{C}([0, T] ; X)$ the space of continuous functions from $[0, T]$ into $X$, the space is Banach for the following norm

$$
\|u\|_{\mathscr{C}([0, T] ; X)}=\sup _{t \in[0, T]}\|u(t)\|_{X}
$$

and by $\mathscr{C}^{k}([0, T] ; X), k \in \mathbb{N}$ the space of $k$ times continuously differentiable functions from $[0, T]$ into $X$, it is a Banach spaces for the following norm

$$
\|u\|_{\mathscr{C}^{k}([0, T] ; X)}=\Sigma_{j=0}^{k}\left\|\frac{d^{j} u}{d t^{j}}\right\|_{\mathscr{C}([0, T] ; X)}
$$

we denote by $\mathscr{C}^{\infty}(\Omega)$ the space of infinitely times continuously differentiable functions on $\Omega$. The space $\mathscr{C}^{\infty}(\Omega)$ of real functions on $\Omega$, with a compact support in $\Omega$, is denoted by $\mathscr{D}(\Omega)$, as in the theory of distributions of L.Schwartz in $([8]), \mathscr{D}^{\prime}(\Omega)$ is the space of distributions on $\Omega$.

We introduce the Sobolev spaces, for $m \in \mathbb{N}, 1 \leq p \leq \infty, W^{m, p}(\Omega)$ is the space of functions $u$ in $L^{p}(\Omega)$ whose distribution derivatives of order $\leq m$ are in $L^{p}(\Omega)$. This is a Banach space for the norm

$$
\|u\|_{W^{m, p}(\Omega)}=\Sigma_{|\alpha| \leq m}\left\|D^{\alpha} u\right\|_{L^{p}(\Omega)}
$$

where

$$
\begin{array}{r}
D^{\alpha} u=\frac{\partial^{\alpha_{1}+\cdots+\alpha_{n}} u}{\partial x_{1}^{\alpha_{1}} \cdots \partial x_{n}^{\alpha_{n}}}, \alpha=\left\{\alpha_{1}, \cdots, \alpha_{n}\right\} \in \mathbb{N}^{n}, \\
|\alpha|=\alpha_{1}+\cdots+\alpha_{n} \text { and } D_{i} u=\frac{\partial u}{\partial x_{i}}, \quad i=1,2, \cdots, n .
\end{array}
$$

When $p=2$ we write $W^{m, 2}(\Omega)=H^{m}(\Omega)$ and this is a Hilbert space for the scalar product

$$
(u, v)_{H^{m}(\Omega)}=\Sigma_{|\alpha| \leq m}\left(D^{\alpha} u, D^{\alpha} v\right) .
$$

we use the space $H_{0}^{1}(\Omega)$

$$
H_{0}^{1}(\Omega)=\text { the closure of } \mathscr{D}(\Omega) \text { in } H^{1}(\Omega)
$$

for study the problem (1) we introduce the space

$$
V=H_{0}^{1}(\Omega)
$$

$V$ is a Hilbert space for the scalar product

$$
(u, v)_{V}=\Sigma_{i=1}^{n}\left(\frac{\partial u}{\partial x_{i}}, \frac{\partial v}{\partial x_{i}}\right)
$$


and that the corresponding norm

$$
\|u\|_{V}=\left\{(u, u)_{V}\right\}^{\frac{1}{2}}
$$

with dual $V^{\prime}=H^{-1}(\Omega)$, the corresponding norm on $V^{\prime}$ is

$$
\|l\|_{*}=\sup _{u \in V} \frac{|\langle l, u\rangle|}{\|u\|_{V}}
$$

for any $l \in V^{\prime}$ and $u \neq 0$, where $\langle.,$.$\rangle is the scalar product between V$ and $V^{\prime}$. To given linear continuous operator $A \in \mathscr{L}\left(V, V^{\prime}\right)$, we can associate a bilinear continuous form $a$ on $V$ by setting

$$
a(u, v)=\langle A u, v\rangle, \forall u, v \in V,
$$

such that $\mathscr{L}\left(V, V^{\prime}\right)$ is the space of linear continuous operators from $V$ into $V^{\prime}$. conversely, to given a bilinear continuous form $a$ on $V$, we can associate with $a$ a linear continuous operator $A$ from $V$ into $V^{\prime}$, and from the properties of $a$ that $A$ is linear continuous, and by the continuity of $a$ if

$$
|a(u, v)| \leq C\|u\|_{V}\|v\|_{V}, C>0, \forall u, v \in V,
$$

then

$$
\|A\|_{*} \leq C
$$

If $a(u, v)=(u, v)_{V}$ is the scalar product of $V$, that $a$ is coercive,

$$
a(u, u) \geq \alpha\|u\|_{V}^{2}, 0 \leq \alpha \leq 1, \forall u \in V .
$$

The Riesz representation theorem to show that each a linear continuous form on $\mathrm{H}$ can be represented with the aid of scalar product. Let $H^{\prime}$ the dual space of $H$, to given $\phi \in H^{\prime}$ there exists a unique $f \in H$ such that the application $\phi \longrightarrow f$ is an isomorphism and isometric that allows us to identify $H$ to the dual space $H^{\prime}$.

In general, but not always, it is also convenient to identify $H$ to its dual $H^{\prime}$. We write position typical where is not place of performance this identification. Let $H=L^{2}(\Omega)$, and $V=H_{0}^{1}(\Omega)$ is a dense in $L^{2}(\Omega)$ since that $V$ it is a Banach space reflexive, we assume that the canonical injection of $V$ in $H$ being continuous, then identify, $H^{\prime} \equiv H$ and $H \subset V^{\prime}$ from the following assertion:

For given $f \in H$, the application $v \in V \longrightarrow(f, v)$ of $V$ into $\mathbb{R}$ is linear continuous on $H$ and by priority on $V$, denote by $T f \in V^{\prime}$ such that

$$
\langle T f, v\rangle=(f, v), \forall f \in H, \forall v \in V
$$

where $T: H \longrightarrow V^{\prime}$ satisfies the following proprieties 
(i) $\|T f\|_{*} \leq C\|f\|$, for any $f \in H$,

(ii) $T$ is injective

(iii) $T(H)$ is a dense in $V^{\prime}$

from $T$, we have $H \subset V^{\prime}$ then we obtain

$$
V \subset H \equiv H^{\prime} \subset V^{\prime}
$$

where each space is dense in the following, the injections being continuous.

We recall some basic results for using in the proof of our main results.

\section{Preliminaries}

Lemma 1. If $u(x, t) \in L^{1}(\Omega \times[0, T])$ satisfies

$$
\int_{\Omega} u(x, t) d x \leq C+B \int_{0}^{t}\left(\int_{\Omega} u(x, s) d x\right) d s, C \in \mathbb{R}, B>0
$$

then

$$
\int_{\Omega} u(x, t) d x \leq C e^{B t}, x \in \Omega, t \in[0, T] .
$$

Proof. we have the inequality

$$
\begin{gathered}
\int_{\Omega} u(x, t) d x \leq C+B \int_{0}^{t}\left(\int_{\Omega} u(x, s) d x\right) d s \leq \\
C+B \int_{0}^{t}\left[C+B \int_{0}^{s}\left(\int_{\Omega} u(x, \sigma) d x\right) d \sigma\right] d s= \\
C(1+B t)+B^{2} \int_{0}^{t}(t-s)\left(\int_{\Omega} u(x, s) d x\right) d s
\end{gathered}
$$

then, we have

$$
\begin{array}{r}
\int_{\Omega} u(x, t) d x \leq C(1+B t)+B^{2} \int_{0}^{t}(t-s)\left(\int_{\Omega} u(x, s) d x\right) d s \leq \\
C(1+B t)+B^{2} \int_{0}^{t}(t-s)\left[C+B\left(\int_{0}^{s}\left(\int_{\Omega} u(x, \sigma) d x\right) d \sigma\right] d s=\right. \\
C\left(1+B t+B^{2} \frac{t^{2}}{2}\right)+B^{3} \int_{0}^{t} \frac{(t-s)^{2}}{2}\left(\int_{\Omega} u(x, s) d x\right) d s
\end{array}
$$


by backward we obtain :

$$
\begin{array}{r}
\int_{\Omega} u(x, t) d x \leq C \\
\left(1+B t+B^{2} \frac{t^{2}}{2}+\cdots+B^{n} \frac{t^{n}}{n !}\right)+ \\
B^{n+1} \int_{0}^{t} \frac{(t-s)^{n}}{n !}\left(\int_{\Omega} u(x, s) d x\right) d s
\end{array}
$$

pass to the limit as $n \longrightarrow+\infty$, we obtain

$$
\int_{\Omega} u(x, t) d x \leq C e^{B t} .
$$

Lemma 2. If $u \in L^{p}(0, T ; X)$ and $\frac{\partial u}{\partial t} \in L^{p}(0, T ; X), 1 \leq p \leq \infty$ then $u$ is a continuous from $[0, T]$ into $X$, almost everywhere on $[0, T]$.

Proof. We find $u$ solution of the problem (1) in the space $L^{\infty}(0, T ; X)$ then we need the derivative $\frac{\partial u}{\partial t}$ in the space $L^{\infty}(0, T ; X)$ we prove $\frac{\partial u}{\partial t} \in L^{p}(0, T ; X)$ if $u \in L^{p}(0, T ; X)$ where $1 \leq p \leq \infty$.

In $([9]), \mathscr{D}^{\prime}(0, T ; X)$ is the space of distributions from $] 0, T[$ into $X$, defined by

$$
\mathscr{D}^{\prime}(0, T ; X)=\mathscr{L}(\mathscr{D}(] 0, T[) ; X) .
$$

if $u \in \mathscr{D}^{\prime}(0, T ; X)$, the distributional derivative is defined by

$$
\frac{\partial u}{\partial t}(\varphi)=-u\left(\frac{d \varphi}{d t}\right), \varphi \in \mathscr{D}(] 0, T[)
$$

if $u \in L^{p}(0, T ; X)$, the corresponding distribution is also defined by $u$ from $] 0, T[$ into $X$, such that

$$
u(\varphi)=\int_{0}^{T} u(t) \varphi(t) d t, \varphi \in \mathscr{D}(] 0, T[)
$$

the integral $u(\varphi) \in X$; we can also defined $\frac{\partial u}{\partial t} \in \mathscr{D}^{\prime}(0, T ; X)$ by $(9)$.

Let $V$ is a Banach space separable and reflexive and $K$ is a closed convex set in $V$.

Theorem 3. We assume that $K$ is a closed convex set unbounded in $V$. Let $A$ is a pseudo-monotone operator from $K$ into $V^{\prime}$, and coercive in the following sense: 
There exists $v_{0} \in K$ such that

$$
\frac{\left\langle A(v), v-v_{0}\right\rangle}{\|v\|_{V}} \longrightarrow+\infty \text { as }\|v\|_{V} \longrightarrow+\infty, v \in K
$$

then, for $f \in V^{\prime}$, there exists $u \in K$ such that

$$
\langle A(u), v-u\rangle \geq\langle f, v-u\rangle, \forall v \in K .
$$

Proof. We note that $A$ is pseudo - monotone from $V$ into $V^{\prime}$ if satisfies the following conditions:

First: $A$ is bounded,

Second: as $j \longrightarrow+\infty, u_{j}$ tending to $u$ weakly in $V$ and

$\lim \sup \left\langle A\left(u_{j}\right), u_{j}-u\right\rangle \leq 0$, then,

$\lim \inf \left\langle A\left(u_{j}\right), u_{j}-v\right\rangle \geq\langle A(u), u-v\rangle$, as $j \longrightarrow+\infty$.

We give the following Theorem

Theorem 4. We assume that $K$ is a convex closed bounded noempty. Let $A$ is a operator pseudo-monotone from $K$ into $V^{\prime}$. Then for $f \in V^{\prime}$, there exists $u$ in $K$ such that

$$
\langle A(u), v-u\rangle \geq\langle f, v-u\rangle, \forall v \in K .
$$

The proof of the Theorem is in ([3], P. 245).

Let

$$
B_{R}=\left\{v \mid v \in V,\|v\|_{V} \leq R\right\}, K_{R}=K \bigcap B_{R},
$$

since $K_{R}$ is a closed convex and bounded, then from Theorem 4, there exists $u_{R} \in K_{R}$ such that

$$
\left\langle A\left(u_{R}\right), v-u_{R}\right\rangle \geq\left\langle f, v-u_{R}\right\rangle \forall v \in K_{R},
$$

choosing $R \geq R_{0}$ such that $\left\|v_{0}\right\|_{V} \leq R_{0}$. Then by taking $v=v_{0}$ in (11) we deduce from (10), that

$$
\left\|u_{R}\right\|_{V} \leq C
$$

and $u_{R}$ is solution of (11), we have $\left\|u_{R}\right\| \leq C$ and if choosing $R>C$, then $u_{R}$ is solution of

$$
\langle A(u), v-u\rangle \geq\langle f, v-u\rangle, \forall v \in K .
$$

indeed, we have $\left\|u_{R}\right\|_{V} \leq C$, then $A\left(u_{R}\right)$ remain in a bounded set of $V^{\prime}$ and there exists a subsequence $R \longrightarrow \infty$ such that

$$
u_{R} \longrightarrow u \text { weakly in } V, A\left(u_{R}\right) \longrightarrow \chi \text { weakly in } V^{\prime},
$$


since $K$ is weakly closed, $u \in K$. We have

$$
\left\langle A\left(u_{R}\right), u_{R}-u\right\rangle \leq\left\langle f, u_{R}-u\right\rangle
$$

on $R \geq\|u\|_{V}=C$, then

$$
\lim \sup \left\langle A\left(u_{R}\right), u_{R}-u\right\rangle \leq 0
$$

and from the pseudo-monotone,

$$
\liminf \left\langle A\left(u_{R}\right), u_{R}-v\right\rangle \geq\langle A(u), u-v\rangle
$$

and since

$$
\left\langle A\left(u_{R}\right), u_{R}-v\right\rangle \leq\left\langle f, u_{R}-v\right\rangle \longrightarrow\langle f, u-v\rangle \forall v \in K,
$$

we deduce from (12) that

$$
\langle A(u), u-v\rangle \leq\langle f, u-v\rangle \forall v \in K,
$$

then we have

$$
\langle A(u), v-u\rangle \geq\langle f, v-u\rangle
$$

\section{Main Results}

\subsection{Existence and Uniqueness of Solutions}

Theorem 5. Assume that $\Omega$ be a bounded open. We give $f, u_{0}, u_{1}$ with

$$
\begin{aligned}
& f \in L^{2}(Q), \\
& u_{0} \in H_{0}^{1}(\Omega), \\
& u_{1} \in L^{2}(\Omega) .
\end{aligned}
$$

There exists a unique solution $u$ satisfies

$$
\begin{gathered}
u \in L^{\infty}\left(0, T ; H_{0}^{1}(\Omega)\right), \\
\frac{\partial u}{\partial t} \in L^{\infty}\left(0, T ; L^{2}(\Omega)\right),
\end{gathered}
$$




$$
\begin{gathered}
\frac{\partial^{2} u}{\partial t^{2}}+\eta \frac{\partial u}{\partial t}-\Delta u=f \text { in } \quad Q, \\
u(0)=u_{0}, \\
\frac{\partial u}{\partial t}(0)=u_{1} .
\end{gathered}
$$

Proof. Proof of the existence. The proof of this Theorem will be made in three steps.

Step 1: The existence is proved by the Faedo - Galerkin method, in ([3]), we take $u^{\prime}=\frac{\partial u}{\partial t}, u^{\prime \prime}=\frac{\partial^{2} u}{\partial t^{2}}$, since the space $V=H_{0}^{1}(\Omega)$ is a separable space. We introduce a sequence of functions $w_{1}, \cdots, w_{m}, \cdots$, such that, $w_{i} \in H_{0}^{1}(\Omega)$, for any $i=1,2, \cdots, m, \cdots$ and for any $m, w_{1}, \cdots, w_{m}$ are linearly independent elements of $H_{0}^{1}(\Omega)$, the finite linear combinations of $w_{i}$ are dense in the space $H_{0}^{1}(\Omega)$.

We find an approximate solution $u_{m}=u_{m}(t)$ of (18) $-(19)-(20)$ as follows

$$
u_{m}(t)=\sum_{i=1}^{m} g_{i m}(t) w_{i},
$$

where $g_{i m}$, are obtained by the conditions

$$
\left\{\begin{array}{r}
\left(u_{m}^{\prime \prime}(t), w_{j}\right)+\eta\left(u_{m}^{\prime}(t), w_{j}\right)+a\left(u_{m}(t), w_{j}\right)=\left(f(t), w_{j}\right), \\
\text { for } j=1, \cdots, m,
\end{array}\right.
$$

where

$$
a(u, v)=\Sigma_{i=1}^{n} \int_{\Omega} \frac{\partial u}{\partial x_{i}} \frac{\partial v}{\partial x_{i}} d x
$$

the system (22) of linear ordinary differential equations is given with the initial conditions, as $m \longrightarrow+\infty$

$$
\begin{aligned}
& u_{m}(0)=u_{0 m}, u_{0 m}=\Sigma_{i=1}^{m} \alpha_{i m} w_{i} \longrightarrow u_{0} \text { in } H_{0}^{1}(\Omega) \\
& u_{m}^{\prime}(0)=u_{1 m}, u_{1 m}=\sum_{i=1}^{m} \beta_{i m} w_{i} \longrightarrow u_{1} \text { in } L^{2}(\Omega)
\end{aligned}
$$

From the linearly independent of $w_{1}, \cdots, w_{m}$, we have $\operatorname{det}\left(w_{i}, w_{j}\right) \neq 0, i=1, \cdots, m$ and $j=1, \cdots, m$ then from the general results on the systems of differential equations, these results guarantees the existence of a solution of $(22)-(24)-(25)$ in the intervalle $[0, t]$. The following a priori estimates show that $t=T$.

Step 2: We multiply (22) by $g_{j m}^{\prime}$, add these relations for $j=1, \cdots, m$, which gives

$$
\left(u_{m}^{\prime \prime}(t), u_{m}^{\prime}(t)\right)+\eta\left(u_{m}^{\prime}(t), u_{m}^{\prime}(t)\right)+a\left(u_{m}(t), u_{m}^{\prime}(t)\right)=
$$




$$
\left(f(t), u_{m}^{\prime}(t)\right)
$$

then

$$
\begin{array}{r}
\frac{1}{2} \frac{d}{d t} \int_{\Omega}\left[\left(u_{m}^{\prime}(t)\right)^{2}+a\left(u_{m}(t), u_{m}(t)\right)\right] d x+\eta \int_{\Omega}\left(u_{m}^{\prime}(t)\right)^{2} d x= \\
\left(f(t), u_{m}^{\prime}(t)\right)
\end{array}
$$

then after an integration on $t$ and using Cauchy-Schwarz inequality, we obtain

$$
\begin{gathered}
\frac{1}{2}\left[\left\|u_{m}^{\prime}(t)\right\|^{2}+\left\|u_{m}(t)\right\|_{V}^{2}\right]+\eta \int_{0}^{t}\left\|u_{m}^{\prime}(\sigma)\right\|^{2} d \sigma \leq \\
\frac{1}{2}\left[\left\|u_{1 m}\right\|^{2}+\left\|u_{0 m}\right\|_{V}^{2}\right]+\int_{0}^{t}\|f(\sigma)\|\left\|u_{m}^{\prime}(\sigma)\right\| d \sigma
\end{gathered}
$$

then

$$
\begin{array}{r}
\frac{1}{2}\left[\left\|u_{m}^{\prime}(t)\right\|^{2}+\left\|u_{m}(t)\right\|_{V}^{2}\right]+\eta \int_{0}^{t}\left\|u_{m}^{\prime}(\sigma)\right\|^{2} d \sigma \leq C+ \\
\frac{1}{2} \int_{0}^{t}\|f(\sigma)\|^{2} d \sigma+\frac{1}{2} \int_{0}^{t}\left\|u_{m}^{\prime}(\sigma)\right\|^{2} d \sigma
\end{array}
$$

where $C>0$ is independent of $m$ from (13) we have

$$
\int_{0}^{T}\|f(\sigma)\|^{2} d \sigma \leq C
$$

we conclude that

$$
\begin{array}{r}
\frac{1}{2}\left[\left\|u_{m}^{\prime}(t)\right\|^{2}+\left\|u_{m}(t)\right\|_{V}^{2}\right]+\eta \int_{0}^{t}\left\|u_{m}^{\prime}(\sigma)\right\|^{2} d \sigma \leq \\
C+\frac{1}{2} \int_{0}^{t}\left\|u_{m}^{\prime}(\sigma)\right\|^{2} d \sigma
\end{array}
$$

we use the Lemma 1, we obtain

$$
\frac{1}{2}\left[\left\|u_{m}^{\prime}(t)\right\|^{2}+\left\|u_{m}(t)\right\|_{V}^{2}\right]+\eta \int_{0}^{t}\left\|u_{m}^{\prime}(\sigma)\right\|^{2} d \sigma \leq C
$$

then if $\eta \geq 0$ we obtain

$$
\left\|u_{m}^{\prime}(t)\right\| \leq C \text { and }\left\|u_{m}\right\|_{V} \leq C
$$


if $\eta<0$ we conclude from (30) that

$$
\frac{1}{2}\left[\left\|u_{m}^{\prime}(t)\right\|^{2}+\left\|u_{m}\right\|_{V}^{2}\right] \leq C+\left(\frac{1}{2}-\eta\right) \int_{0}^{t}\left\|u_{m}^{\prime}(\sigma)\right\|^{2} d \sigma
$$

we use the Lemma 1, we obtain

$$
\frac{1}{2}\left[\left\|u_{m}^{\prime}(t)\right\|^{2}+\left\|u_{m}(t)\right\|_{V}^{2}\right] \leq C
$$

then we obtain

$$
\left\|u_{m}^{\prime}(t)\right\| \leq C \text { and }\left\|u_{m}\right\|_{V} \leq C
$$

then we conclude that $t=T$, from (32) - (33) we obtain the result, letting $m \longrightarrow+\infty$,

$$
\begin{array}{r}
u_{m} \text { remain in a bounded set of } L^{\infty}\left(0, T ; H_{0}^{1}(\Omega)\right) \\
\text { and } u_{m}^{\prime} \text { remain in a bounded set of } L^{\infty}\left(0, T ; L^{2}(\Omega)\right)
\end{array}
$$

\section{Step 3: Pass to the limit}

From Dunford-pettis theorem in $([16])$ to show that the space $L^{\infty}\left(0, T ; H_{0}^{1}(\Omega)\right.$ be a given with dual $L^{1}\left(0, T ; H^{-1}(\Omega)\right)$ and the space $L^{\infty}\left(0, T ; L^{2}(\Omega)\right)$ be a given with dual $L^{1}\left(0, T ; L^{2}(\Omega)\right)$ by a consequence there exists a subsequence $u_{\mu}$ of $u_{m}$ such that

$$
\begin{gathered}
u_{\mu} \longrightarrow u \text { weakly star in } L^{\infty}\left(0, T ; H_{0}^{1}(\Omega)\right) \\
\text { and } u_{\mu}^{\prime} \longrightarrow u^{\prime} \text { weakly star in } L^{\infty}\left(0, T ; L^{2}(\Omega)\right)
\end{gathered}
$$

from (34) to show that $u_{m}$ is a bounded in $L^{2}\left(0, T ; H_{0}^{1}(\Omega)\right)$ and $u_{m}^{\prime}$ is a bounded in $L^{2}\left(0, T ; L^{2}(\Omega)\right)$. Then to show that $u_{m}$ remain in a bounded set of $H^{1}(Q)$. Then from Rellich-Kondrachoff theorem in ([4]) to show that

$$
\text { the injection of } H^{1}(Q) \text { in } L^{2}(Q) \text { is compact }
$$

we assume that subsequence $u_{\mu}$ of $u_{m}$ satisfies (35) - (36)

$$
u_{\mu} \longrightarrow u \text { strongly in } L^{2}(\Omega) \text { and almost everywhere }
$$

pass to the limit in (22) and using for $m=\mu$, let $j$ is a fixed and $\mu>j$, then from $(22)$

$$
\left(u_{\mu}^{\prime \prime}, w_{j}\right)+\eta\left(u_{\mu}^{\prime}, w_{j}\right)+a\left(u_{\mu}, w_{j}\right)=\left(f, w_{j}\right)
$$

from $(35)-(36)$ we have

$$
a\left(u_{\mu}, w_{j}\right) \longrightarrow a\left(u, w_{j}\right) \text { weakly star in } L^{\infty}\left(0, T ; H_{0}^{1}(\Omega)\right),
$$




$$
\left(u_{\mu}^{\prime}, w_{j}\right) \longrightarrow\left(u^{\prime}, w_{j}\right) \text { weakly star in } L^{\infty}\left(0, T ; L^{2}(\Omega)\right)
$$

and

$$
\left(u_{\mu}^{\prime \prime}, w_{j}\right)=\frac{d}{d t}\left(u_{\mu}^{\prime}, w_{j}\right) \longrightarrow\left(u^{\prime \prime}, w_{j}\right) \text { in } \mathscr{D}^{\prime}(0, T)
$$

we conclude from (37) that

$$
\frac{d^{2}}{d t^{2}}\left(u, w_{j}\right)+\eta \frac{d}{d t}\left(u, w_{j}\right)+a\left(u, w_{j}\right)=\left(f, w_{j}\right)
$$

this for $j$ is a fixed arbitrary. We multiply (38) by $g_{j m}$, add these relation for $j=1, \cdots, m$ we conclude that

$$
\frac{d^{2}}{d t^{2}}(u, v)+\eta \frac{d}{d t}(u, v)+a(u, v)=(f, v), \forall v \in V
$$

then $u$ satisfies (18) and (17) - (16). For show that (19) is satisfying, from $(35)-(36)$ and the Lemma 2 we have, $u_{\mu}(0) \longrightarrow u(0)$ weakly in $L^{2}(\Omega)$, and from $(24), u_{\mu}(0)=u_{0 \mu} \longrightarrow u_{0}$ in $H_{0}^{1}(\Omega)$, then we have (19). For show that $(20)$ is satisfying we prove the Lemma

Lemma 6. Let $Q$ be a bounded open set in $\mathbb{R}_{x}^{n} \times \mathbb{R}_{t}, u_{\mu}$ and $u$ are functions in $L^{q}(Q), 1<q<\infty$, such that

$$
\left\|u_{\mu}\right\|_{L^{q}(Q)} \leq C, u_{\mu} \longrightarrow u \text { almost everywhere in } Q
$$

then $u_{\mu} \longrightarrow u$ weakly in $L^{q}(Q)$.

Proof. Suppose that on a measurable set $E$, we note that

$1 \leq p \leq+\infty, 1 \leq q \leq+\infty$ are conjugated, $\frac{1}{p}+\frac{1}{q}=1$, let $v_{\mu}$ be a sequence of $L^{p}(E)$, tending to $v$, weakly in $L^{p}(E)$, as $\mu \longrightarrow+\infty$, if $\lim \int_{E} v_{\mu} \xi d x=\int_{E} v \xi d x$, for any $\xi \in L^{p}(E)$.

Let $N$ is an increasing sequence tending to $+\infty$, we introduce

$$
E_{N}=\left\{(x, t)|(x, t) \in Q,| u_{\mu}(x, t)-u(x, t) \mid \leq 1, \text { for } \mu \geq N\right\}
$$

$E_{N}$ are measurable set increases with $N$ and measure $\left(E_{N}\right) \longrightarrow$ measure $(Q)$, as $N \longrightarrow+\infty$. Let $\Phi_{N}$ the set of functions $\varphi$ in $L^{q^{\prime}}(Q)$, such that $L^{q^{\prime}}(Q)$ denote the conjugate space of $L^{q}(Q)$,

$\frac{1}{q}+\frac{1}{q}=1$, with a support in $E_{N}$ and let $\Phi=\bigcup_{N \longrightarrow+\infty} \Phi_{N}, \Phi$ is dense in $L^{q}(Q)$. If we take $\varphi \in \Phi$, then from Lebesgue dominated convergence theorem we obtain

$$
\int_{0}^{T} \int_{\Omega} \varphi(x, t)\left(u_{\mu}(x, t)-u(x, t)\right) d x d t \longrightarrow 0, \text { as } \mu \longrightarrow+\infty
$$


indeed, we have $u_{\mu}$ and $u$ are functions in $L^{q}(Q), 1<q<\infty$, such that $\left\|u_{\mu}\right\|_{L^{q}(Q)} \leq C, u_{\mu} \longrightarrow u$ almost everywhere in $Q$, since $\left|\varphi(x, t)\left(u_{\mu}(x, t)-u(x, t)\right)\right| \leq|\varphi(x, t)|$, and $\varphi \in \Phi_{N_{0}}$, we take $\mu \geq N_{0}$, then $\varphi(x, t)\left(u_{\mu}(x, t)-u(x, t)\right) \longrightarrow 0$ almost everywhere, as $\Phi$ is dense in $L^{q^{\prime}}(Q)$, then $\int_{0}^{T} \int_{\Omega} \varphi(x, t) u_{\mu}(x, t) d x d t \longrightarrow$ $\int_{0}^{T} \int_{\Omega} \varphi(x, t) u(x, t) d x d t$, as $\mu \longrightarrow+\infty$, then $u_{\mu} \longrightarrow u$ weakly in $L^{q}(Q)$.

From (40),

$$
\left(u_{\mu}^{\prime \prime}, w_{j}\right) \longrightarrow\left(u^{\prime \prime}, w_{j}\right), \text { weakly star in } L^{\infty}\left(0, T ; L^{2}(\Omega)\right)
$$

then from Lemma 2 with $X=\mathbb{R}$

$$
\left.\left(u_{\mu}^{\prime}(0), w_{j}\right) \longrightarrow\left(u^{\prime}, w_{j}\right)\right|_{t=0}=\left(u^{\prime}(0), w_{j}\right)
$$

and from (25),

$$
\left(u_{\mu}^{\prime}(0), w_{j}\right) \longrightarrow\left(u_{1}, w_{j}\right),
$$

then

$$
\left(u^{\prime}(0), w_{j}\right)=\left(u_{1}, w_{j}\right),
$$

for any $j$, then we have (20).

Remark 7. From (2) and (3), $u=0$ on $\Sigma$, then the condition $u=0$ on $\Sigma$ is satisfies in (16).

In ([4]) from (16) - (17) and Lemma 2, we obtain that $u$ is continuous from $[0, T]$ into $L^{2}(\Omega)$ then (19) has a sense. To verify that (20) has a sense, using the equation (18) can be written as

$$
\frac{\partial^{2} u}{\partial t^{2}}=f+\Delta u-\eta \frac{\partial u}{\partial t}
$$

since $\Delta \in \mathscr{L}\left(H_{0}^{1}(\Omega), H^{-1}(\Omega)\right)$, we have $\Delta u \in L^{\infty}\left(0, T ; H^{-1}(\Omega)\right)$ from (13) (17) we have $f \in L^{2}\left(0, T ; L^{2}(\Omega)\right), \frac{\partial u}{\partial t} \in L^{\infty}\left(0, T ; L^{2}(\Omega)\right.$

from (41) we obtain that

$$
\frac{\partial^{2} u}{\partial t^{2}} \in L^{2}\left(0, T ; L^{2}(\Omega)\right)+L^{\infty}\left(0, T ; H^{-1}(\Omega)+L^{2}(\Omega)\right)
$$

then

$$
\frac{\partial^{2} u}{\partial t^{2}} \in L^{2}\left(0, T ; H^{-1}(\Omega)+L^{2}(\Omega)\right)
$$

since $\frac{\partial u}{\partial t} \in L^{\infty}\left(0, T ; L^{2}(\Omega)\right)$, and from Lemma 2 , that $\frac{\partial u}{\partial t}$ is continuous from $[0, T]$ into $H^{-1}(\Omega)+L^{2}(\Omega)$, such that $(20)$ has a sense. 


\section{Proof of the Uniqueness}

Theorem 8. The equations defined by (18) - (19) - (20) in Theorem 5, has a unique solution $u$.

Proof. Assume that $u, v$ are two solutions of $(18)-(19)-(20)$, then $w=u-v$ satisfies

$$
\begin{aligned}
& \frac{\partial^{2} w}{\partial t^{2}}+\eta \frac{\partial w}{\partial t}-\Delta w=0 \\
& w(0)=0, \quad w^{\prime}(0)=0 \\
& w \in L^{\infty}\left(0, T ; H_{0}^{1}(\Omega)\right), \\
& w^{\prime} \in L^{\infty}\left(0, T ; L^{2}(\Omega)\right) .
\end{aligned}
$$

by taking $w^{\prime}=\frac{\partial w}{\partial t}, w^{\prime \prime}=\frac{\partial^{2} w}{\partial t^{2}}$

we multiply (42) by $w^{\prime}$, we obtain that

$$
\left(w^{\prime \prime}, w^{\prime}\right)+\eta\left(w^{\prime}, w^{\prime}\right)-\left(\Delta w, w^{\prime}\right)=0,
$$

by using integration by parts and (43), we obtain

$$
\frac{d}{d t}\left[\left\|w^{\prime}(t)\right\|^{2}+\|w(t)\|_{V}^{2}\right]+2 \eta\left\|w^{\prime}(t)\right\|^{2}=0
$$

integration from 0 to $t$ and from (43) we obtain

$$
\left\|w^{\prime}(t)\right\|^{2}+\|w(t)\|_{V}^{2}+2 \eta \int_{0}^{t}\left\|w^{\prime}(\sigma)\right\|^{2} d \sigma=0
$$

if $\eta \geq 0$, we obtain that

$$
\left\|w^{\prime}(t)\right\|^{2}+\|w(t)\|_{V}^{2} \leq 0
$$

and $w(t)=0, \forall t \in[0, T]$

if $\eta<0$, we write

$$
\left\|w^{\prime}(t)\right\|^{2}+\|w(t)\|_{V}^{2} \leq-2 \eta \int_{0}^{t}\left(\left\|w^{\prime}(\sigma)\right\|^{2}+\|w(\sigma)\|_{V}^{2}\right) d \sigma
$$

using the Lemma 1, we obtain (49), then $w(t)=0, \forall t \in[0, T]$.

To justify the previous conclusion we use Method in linear hyperbolic equations. Let $s \in] 0, T]$, we introduce

$$
\psi(t)=\left\{\begin{array}{r}
-\int_{t}^{s} w(\sigma) d \sigma, t \leq s \\
0, t>s
\end{array}\right.
$$


and $\psi(t)=w_{1}(t)-w_{1}(s)$ if $t \leq s$ such that $w_{1}(t)=\int_{0}^{t} w(\sigma) d \sigma$. We multiply (42) by $\psi(t)$ we obtain

$$
\int_{0}^{s}\left(w^{\prime \prime}, \psi\right) d t+\eta \int_{0}^{s}\left(w^{\prime}, \psi\right) d t-\int_{0}^{s}(\Delta w, \psi) d t=0
$$

by using integration by parts and (43), we obtain

$$
-\int_{0}^{s}\left(w^{\prime}, \psi^{\prime}\right) d t+\eta \int_{0}^{s}\left(w^{\prime}, \psi\right) d t+\int_{0}^{s} a(w, \psi) d t=0
$$

then, since $\psi^{\prime}=w$ and $\psi(0)=-w_{1}(s)$

$$
-\frac{1}{2}\|w(s)\|^{2}-\eta \int_{0}^{s}\left\|\psi^{\prime}\right\|^{2} d t-\frac{1}{2}\left\|w_{1}(s)\right\|_{V}^{2}=0
$$

then

$$
\frac{1}{2}\|w(s)\|^{2}+\eta \int_{0}^{s}\left\|\psi^{\prime}\right\|^{2} d t+\frac{1}{2}\left\|w_{1}(s)\right\|_{V}^{2}=0
$$

if $\eta \geq 0$, we obtain

$$
\|w(s)\|^{2}+\left\|w_{1}(s)\right\|_{V}^{2} \leq 0
$$

and $w(t)=0$ for $t \in[0, T]$

if $\eta<0$, we write

$$
\|w(s)\|^{2}+\left\|w_{1}(s)\right\|_{V}^{2} \leq-2 \eta \int_{0}^{s}\left(\left\|\psi^{\prime}(t)\right\|^{2}+\left\|w_{1}(t)\right\|_{V}^{2}\right) d t
$$

using the Lemma 1, we obtain $(50)$, then $w(t)=0$ for $t \in[0, T]$.

Remark 9. In the case of the uniqueness the sequence $u_{m}$ of approximate solutions converges to $u$.

\subsection{A Regularity Result}

Theorem 10. The hypotheses are those of theorem 5 with another

$$
\begin{gathered}
\frac{\partial f}{\partial t} \in L^{2}(Q), \\
u_{0} \in H_{0}^{1}(\Omega) \bigcap H^{2}(\Omega), \\
u_{1} \in H_{0}^{1}(\Omega),
\end{gathered}
$$


then there exists a unique solution $u$ of $(18)-(19)-(20)$ satisfies

$$
\begin{gathered}
u \in L^{\infty}\left(0, T ; H_{0}^{1}(\Omega) \bigcap H^{2}(\Omega)\right), \\
u^{\prime} \in L^{\infty}\left(0, T ; H_{0}^{1}(\Omega)\right), \\
u^{\prime \prime} \in L^{\infty}\left(0, T ; L^{2}(\Omega)\right) .
\end{gathered}
$$

\section{Proof. Proof of the Existence}

The Proof of this Theorem will be made in two steps, the existence is proved by the Faedo-Galerkin method, in ([3]).

From the approximate solution $u_{m}$ of $(22)-(24)-(25)$, we take $w_{j}, j=$ $1,2, \cdots, m$ is a basis in the space $H_{0}^{1}(\Omega) \bigcap H^{2}(\Omega)$,

from $(24)-(25)$ we assume that

$$
\begin{gathered}
u_{0 m} \longrightarrow u_{0} \text { in } H_{0}^{1}(\Omega) \bigcap H^{2}(\Omega), \\
u_{1 m} \longrightarrow u_{1} \text { in } H_{0}^{1}(\Omega) .
\end{gathered}
$$

We prove in step 1 an additional a priori estimate to show that the existence of a solution with $(55)-(56)$, and we prove (54) in step 2 by using the equation (18).

Step 1: We deduce from (22) that

$$
\left(u_{m}^{\prime \prime}(0)+\eta u_{m}^{\prime}(0), w_{j}\right)=\left(f(0)+\Delta u_{0 m}, w_{j}\right), j=1, \cdots, m
$$

from (51) and Lemma 2, $f(0) \in L^{2}(\Omega)$, and from (57)

$$
\left\|\Delta u_{0 m}\right\|=\left(\int_{\Omega}\left(\Delta u_{0 m}\right)^{2} d x\right)^{\frac{1}{2}}<C,
$$

we multiply (59) by $g_{j m}^{\prime \prime}(0)$ and add these relations for $j=1, \cdots, m$ which gives

$$
\int_{\Omega}\left(u_{m}^{\prime \prime}(0)\right)^{2} d x=\left(f(0)+\Delta u_{0 m}, u_{m}^{\prime \prime}(0)\right)-\eta\left(u_{m}^{\prime}(0), u_{m}^{\prime \prime}(0)\right)
$$

by using the Cauchy-Schwarz inequality, we obtain

$$
\begin{array}{r}
\int_{\Omega}\left(u_{m}^{\prime \prime}(0)\right)^{2} d x \leq\left[\left(\int_{\Omega}(f(0))^{2} d x\right)^{\frac{1}{2}}+\left(\int_{\Omega}\left(\Delta u_{0 m}\right)^{2} d x\right)^{\frac{1}{2}}+\right. \\
\left.\eta\left(\int_{\Omega}\left(u_{m}^{\prime}(0)\right)^{2} d x\right)^{\frac{1}{2}}\right]\left(\int_{\Omega}\left(u_{m}^{\prime \prime}(0)\right)^{2} d x\right)^{\frac{1}{2}}
\end{array}
$$


then we conclude by using $(57)-(58)$ and $f(0) \in L^{2}(\Omega)$ that

$$
\left(\int_{\Omega}\left(u_{m}^{\prime \prime}(0)\right)^{2} d x\right)^{\frac{1}{2}} \leq C .
$$

by differentiating (22) with respect to $t$, we obtain

$$
\left\{\begin{array}{r}
\left(u_{m}^{\prime \prime \prime}(t), w_{j}\right)+\eta\left(u_{m}^{\prime \prime}(t), w_{j}\right)+a\left(u_{m}^{\prime}(t), w_{j}\right)=\left(f^{\prime}(t), w_{j}\right) \\
\text { for } j=1, \cdots, m
\end{array}\right.
$$

we multiply $(61)$ by $g_{j m}^{\prime \prime}(t)$, add these relations for $j=1, \cdots, m$, we obtain

$$
\left(u_{m}^{\prime \prime \prime}(t), u_{m}^{\prime \prime}(t)\right)+\eta\left(u_{m}^{\prime \prime}(t), u_{m}^{\prime \prime}(t)\right)+a\left(u_{m}^{\prime}(t), u_{m}^{\prime \prime}(t)\right)=\left(f^{\prime}(t), u_{m}^{\prime \prime}(t)\right)
$$

then

$$
\frac{1}{2} \frac{d}{d t} \int_{\Omega}\left[\left(u_{m}^{\prime \prime}(t)\right)^{2}+\left\|u_{m}^{\prime}(t)\right\|_{V}^{2}\right] d x+\eta \int_{\Omega}\left(u_{m}^{\prime \prime}(t)\right)^{2} d x=\left(f^{\prime}(t), u_{m}^{\prime \prime}(t)\right)
$$

integrating from 0 to $t$ and using Cauchy-Schwarz inequality we obtain

$$
\begin{array}{r}
\frac{1}{2} \int_{\Omega}\left[\left(u_{m}^{\prime \prime}(t)\right)^{2}+\left\|u_{m}^{\prime}(t)\right\|_{V}^{2}\right] d x+\eta \int_{0}^{t}\left(\int_{\Omega}\left(u^{\prime \prime}(\sigma)\right)^{2} d x\right) d \sigma \leq \\
\frac{1}{2} \int_{\Omega}\left[\left(u_{m}^{\prime \prime}(0)\right)^{2}+\left\|u_{m}^{\prime}(0)\right\|_{V}^{2}\right] d x+\int_{0}^{t}\left\|f^{\prime}(\sigma)\right\|\left\|u_{m}^{\prime \prime}(\sigma)\right\| d \sigma \leq \\
\frac{1}{2} \int_{\Omega}\left[\left(u_{m}^{\prime \prime}(0)\right)^{2}+\left\|u_{m}^{\prime}(0)\right\|_{V}^{2}\right] d x+\frac{1}{2} \int_{0}^{t}\left(\int_{\Omega}\left(f^{\prime}(\sigma)\right)^{2} d x\right) d \sigma+ \\
\frac{1}{2} \int_{0}^{t}\left(\int_{\Omega}\left(u_{m}^{\prime \prime}(\sigma)\right)^{2} d x\right) d \sigma
\end{array}
$$

by using $(51)-(58)-(60)$, we obtain

$$
\begin{array}{r}
\frac{1}{2}\left[\left\|u_{m}^{\prime \prime}(t)\right\|^{2}+\left\|u_{m}^{\prime}\right\|_{V}^{2}\right]+\eta \int_{0}^{t}\left(\int_{\Omega}\left(u_{m}^{\prime \prime}(\sigma)\right)^{2} d x\right) d \sigma \leq \\
C+\frac{1}{2} \int_{0}^{t}\left(\int_{\Omega}\left(u_{m}^{\prime \prime}(\sigma)\right)^{2} d x\right) d \sigma
\end{array}
$$

we use the Lemma 1, we obtain

$$
\frac{1}{2} \int_{\Omega}\left[\left(u_{m}^{\prime \prime}(t)\right)^{2}+\left\|u_{m}^{\prime}(t)\right\|_{V}^{2}\right] d x+\eta \int_{0}^{t}\left(\int_{\Omega}\left(u_{m}^{\prime \prime}(\sigma)\right)^{2} d x\right) d \sigma \leq C
$$


then if $\eta \geq 0$ we obtain

$$
\left\|u_{m}^{\prime}\right\|_{V} \leq C \text { and }\left\|u_{m}^{\prime \prime}\right\| \leq C
$$

if $\eta<0$ from (62) we obtain

$$
\frac{1}{2}\left[\left\|u_{m}^{\prime \prime}(t)\right\|^{2}+\left\|u_{m}^{\prime}(t)\right\|_{V}^{2}\right] \leq C+\left(\frac{1}{2}-\eta\right) \int_{0}^{t}\left(\int_{\Omega}\left(u_{m}^{\prime \prime}(\sigma)\right)^{2} d x\right) d \sigma
$$

we use the Lemma 1 we obtain

$$
\frac{1}{2}\left[\left\|u_{m}^{\prime \prime}(t)\right\|^{2}+\left\|u_{m}^{\prime}\right\|_{V}^{2}\right] \leq C
$$

then we obtain

$$
\left\|u_{m}^{\prime}\right\|_{V} \leq C \text { and }\left\|u_{m}^{\prime \prime}(t)\right\| \leq C
$$

then from $(64)-(66)$ we obtain the result

$$
\begin{gathered}
u_{m}^{\prime} \text { remain in a bounded set of } L^{\infty}\left(0, T ; H_{0}^{1}(\Omega)\right), \\
u_{m}^{\prime \prime} \text { remain in a bounded set of } L^{\infty}\left(0, T ; L^{2}(\Omega)\right) .
\end{gathered}
$$

Then there exists a subsequence $u_{\mu}$ of $u_{m}$ as in the proof of the existence Theorem 5 such that, $u$ satisfies $(55)-(56)$, then we have $u \in L^{\infty}\left(0, T ; H_{0}^{1}(\Omega)\right)$ from the theorem 5 , for proved (54), to verify that

$$
u \in L^{\infty}\left(0, T ; H^{2}(\Omega)\right)
$$

Step 2: We prove (67).

We conclude from (18) that

$$
\Delta u=u^{\prime \prime}+\eta u^{\prime}-f
$$

from (13) and (51), $f \in L^{\infty}\left(0, T ; L^{2}(\Omega)\right)$ and with (56) we conclude from (68) that

$$
\Delta u \in L^{\infty}\left(0, T ; L^{2}(\Omega)\right)
$$

put

$$
\Delta u=h,
$$

since $\Delta: H_{0}^{1}(\Omega) \longrightarrow H^{-1}(\Omega)$ is an isomorphism with continuous inverse $G=$ $\Delta^{-1}$, and since $u \in L^{\infty}\left(0, T ; H_{0}^{1}(\Omega)\right)$ we have

$$
u(t)=G h(t) \text { almost everywhere }
$$


in $([4],[6])$ we have the theorems for regularity of solutions of linear elliptic equations are given

$$
G \in \mathscr{L}\left(L^{2}(\Omega), H^{2}(\Omega)\right)
$$

then from (69) - (70), we obtain

$$
u \in L^{\infty}\left(0, T ; H^{2}(\Omega)\right) .
$$

\section{Proof of the Uniqueness}

Theorem 11. The solution $u$ in the theorem 10 is a unique.

Proof. Assume that $u, v$ are two solutions given in the theorem 10, then $w=u-v$ satisfies

$$
\begin{gathered}
\frac{\partial^{2} w}{\partial t^{2}}+\eta \frac{\partial w}{\partial t}-\Delta w=0 \\
w(0)=0, \quad w^{\prime}(0)=0, \\
w \in L^{\infty}\left(0, T ; H_{0}^{1}(\Omega) \bigcap H^{2}(\Omega)\right), \\
w^{\prime} \in L^{\infty}\left(0, T ; H_{0}^{1}(\Omega)\right) \\
w^{\prime \prime} \in L^{\infty}\left(0, T ; L^{2}(\Omega)\right)
\end{gathered}
$$

we multiply (71) by $w^{\prime}$, we obtain

$$
\left(w^{\prime \prime}, w^{\prime}\right)+\eta\left(w^{\prime}, w^{\prime}\right)-\left(\Delta w, w^{\prime}\right)=0
$$

by integration by parts and (72), we obtain

$$
\frac{1}{2} \frac{d}{d t} \int_{\Omega}\left[\left(w^{\prime}(t)\right)^{2}+\|w(t)\|_{V}^{2}\right] d x+\eta \int_{\Omega}\left(w^{\prime}(t)\right)^{2} d x=0
$$

integration from 0 to $t$ and from (72) we obtain

$$
\frac{1}{2} \int_{\Omega}\left[\left(w^{\prime}(t)\right)^{2}+\|w(t)\|_{V}^{2}\right] d x+\eta \int_{0}^{t}\left(\int_{\Omega}\left(w^{\prime}(\sigma)\right)^{2} d x\right) d \sigma=0
$$

if $\eta \geq 0$, we obtain that

$$
\int_{\Omega}\left[\left(w^{\prime}(t)\right)^{2}+\|w(t)\|_{V}^{2}\right] d x \leq 0
$$


then $w(t)=0$, for $t \in[0, T]$

if $\eta<0$, we write

$$
\int_{\Omega}\left[\left(w^{\prime}(t)\right)^{2}+\|w(t)\|_{V}^{2}\right] d x \leq-2 \eta \int_{0}^{t}\left(\int_{\Omega}\left[\left(w^{\prime}(\sigma)\right)^{2}+\|w(\sigma)\|_{V}^{2}\right] d x\right) d \sigma
$$

using the Lemma 1, we obtain (76), then $w(t)=0$ for $t \in[0, T]$.

For justify the previous conclusion the uniqueness applying the same argument in uniqueness Theorem 8 , then the sequence $u_{m}$ of approximate solutions converges to $u$.

\subsection{Semi Discretization and Variational inequalities}

In ([3], P. 432), we apply semi-discretization in time, to establish the existence and uniqueness of solution, then

we give the following Theorem.

Such that from (8) we have

$$
V \subset H \equiv H^{\prime} \subset V^{\prime}
$$

and from (7), we have

$$
-\Delta \in \mathscr{L}\left(V, V^{\prime}\right),\langle-\Delta v, v\rangle \geq \alpha\|v\|_{V}^{2}, 0 \leq \alpha \leq 1, v \in V .
$$

Theorem 12. We assume that (77) - (78) are satisfied.

Let $K$ is a closed convex set in $V$. We give

$$
\begin{gathered}
f \in L^{2}(0, T ; H), \\
u_{0} \in K, \\
u_{1} \in H .
\end{gathered}
$$

There exists a unique solution $u$, such that

$$
\begin{gathered}
u \in \mathscr{C}([0, T] ; V) \bigcap \mathscr{C}^{1}([0, T] ; H) . \\
u(t) \in K \text {, almost everywhere, } u^{\prime}(t) \in H .
\end{gathered}
$$

And in the cases 1.,2. We obtain the inequality

$$
\int_{0}^{T}\left\langle u^{\prime \prime}(t)+\eta u^{\prime}(t)-\Delta u(t)-f(t), v(t)-u(t)\right\rangle d t+
$$




$$
\left\|v^{1}-u^{1}\right\|^{2}+\Sigma_{n=1}^{N-1}\left\|v^{n-1}-u^{n-1}-\left(v^{n}-u^{n}\right)\right\|^{2} \geq 0
$$

$\forall v \in \mathscr{C}([0, T] ; V), v^{\prime} \in \mathscr{C}([0, T] ; H), v^{\prime \prime} \in L^{2}\left(0, T, V^{\prime}\right)$, $v(t) \in K$, almost everywhere, $v^{\prime}(t) \in H$.

And in the cases 2., 3. We obtain the inequality

$$
\begin{array}{r}
\int_{0}^{T}\left\langle u^{\prime \prime}(t)+\eta u^{\prime}(t)-\Delta u(t)-f(t), v(t)-u(t)\right\rangle d t+ \\
\Sigma_{n=1}^{N-1}\left\|v^{n+1}-u^{n+1}-\left(v^{n}-u^{n}\right)\right\|^{2}+ \\
\Sigma_{n=1}^{N-1}\left\|v^{n-1}-u^{n-1}-\left(v^{n}-u^{n}\right)\right\|^{2} \geq 0
\end{array}
$$

$\forall v \in \mathscr{C}([0, T] ; V), v^{\prime} \in \mathscr{C}([0, T] ; H), v^{\prime \prime} \in L^{2}\left(0, T ; V^{\prime}\right)$, $v(t) \in K$, almost everywhere, $v^{\prime}(t) \in H$.

And in the cases 2.,4.,5. We obtain the inequality

$$
\begin{array}{r}
\int_{0}^{T}\left\langle u^{\prime \prime}(t)+\eta u^{\prime}(t)-\Delta u(t)-f(t), v(t)-u(t)\right\rangle d t+ \\
\left(\frac{1+\eta k}{2}\right)\left\|v^{1}-u^{1}\right\|^{2}+ \\
\left(\frac{1+\eta k}{2}\right) \Sigma_{n=1}^{N-1}\left\|v^{n+1}-u^{n+1}-\left(v^{n}-u^{n}\right)\right\|^{2}+ \\
\Sigma_{n=1}^{N-1}\left\|v^{n-1}-u^{n-1}-\left(v^{n}-u^{n}\right)\right\|^{2} \geq 0
\end{array}
$$

$\forall v \in \mathscr{C}([0, T] ; V), v^{\prime} \in \mathscr{C}([0, T] ; H), v^{\prime \prime} \in L^{2}\left(0, T ; V^{\prime}\right)$, $v(t) \in K$, almost everywhere, $v^{\prime}(t) \in H$.

Proof. Semi-discretization in time We introduce

$$
k=\triangle t=\frac{T}{N}
$$

$N$ is an integer fixed in $\mathbb{N}$ and $u^{n}$ an approximation to $u$ at time $n k$. We introduce

$$
f^{n}=\frac{1}{k} \int_{n k}^{(n+1) k} f(\sigma) d \sigma, n \geq 1 ;
$$

we take

$$
u^{0}=u_{0}
$$

and we define $u_{n}$ by

$$
\left\{\begin{array}{r}
\left(\frac{u^{n+1}-2 u^{n}+u^{n-1}}{k^{2}}+\eta \frac{u^{n+1}-u^{n}}{k}, v-u^{n}\right)+ \\
\left\langle-\Delta u^{n}-f^{n}, v-u^{n}\right\rangle \geq 0 \\
\forall v \in K, \forall v^{\prime} \in H, u^{n} \in K, n=1, \cdots, N-1
\end{array}\right.
$$


The system (90) is an elliptic variational inequality, has a unique solution. Indeed, (90) is equivalent to, for any $v \in K, v^{\prime} \in H$

$$
\left\langle-\Delta u^{n}-\left(\frac{2}{k^{2}}+\frac{\eta}{k}\right) u^{n}, v-u^{n}\right\rangle \geq\left(f^{n}-\left(\frac{1}{k^{2}}+\frac{\eta}{k}\right) u^{n+1}-\frac{1}{k^{2}} u^{n-1}, v-u^{n}\right),
$$

then by applying the Theorem 3 , to the operator

$$
-\Delta-\left(\frac{2}{k^{2}}+\frac{\eta}{k}\right) I .
$$

Where $I$ is the identity operator, then we prove that operator $-\Delta-\left(\frac{2}{k^{2}}+\frac{\eta}{k}\right) I$ is coercive, we have

$\left\langle-\Delta v, v_{0}\right\rangle=a\left(v, v_{0}\right)=\Sigma_{i=1}^{N} \int_{\Omega} \frac{\partial v}{\partial x_{i}} \frac{\partial v_{0}}{\partial x_{i}} d x, v_{0} \in K$, and from (4) - (7) we obtain that

$$
\begin{array}{r}
\left\langle-\left(\Delta+\left(\frac{2}{k^{2}}+\frac{\eta}{k}\right)\right) v, v-v_{0}\right\rangle= \\
\langle-\Delta v, v\rangle-\left\langle-\Delta v, v_{0}\right\rangle-\left(\left(\frac{2}{k^{2}}+\frac{\eta}{k}\right) v, v-v_{0}\right) \geq \\
\alpha\|v\|_{V}^{2}-a\left(v, v_{0}\right)-\left(\left(\frac{2}{k^{2}}+\frac{\eta}{k}\right) v, v-v_{0}\right)
\end{array}
$$

then for any $v \in K$

$$
\begin{aligned}
\left\langle-\left(\Delta+\left(\frac{2}{k^{2}}+\frac{\eta}{k}\right)\right) v, v-v_{0}\right\rangle & \longrightarrow+\infty \\
\|v\|_{V} & \text { as }\|v\|_{V} \longrightarrow+\infty
\end{aligned}
$$

and we prove that operator $-\Delta-\left(\frac{2}{k^{2}}+\frac{\eta}{k}\right) I$ is pseudo monotone, first we prove that operator is bounded then for any $u, v \in K$, from (5) we have

$$
\begin{array}{r}
\left\langle-\Delta u-\left(\frac{2}{k^{2}}+\frac{\eta}{k}\right) u, v\right\rangle=\langle-\Delta u, v\rangle-\left(\frac{2}{k^{2}}+\frac{\eta}{k}\right)(u, v) \leq \\
C\|u\|_{V}\|v\|_{V}-\left(\frac{2}{k^{2}}+\frac{\eta}{k}\right)(u, v) \leq C\|u\|_{V}\|v\|_{V}
\end{array}
$$

then

$$
\left\langle-\Delta u-\left(\frac{2}{k^{2}}+\frac{\eta}{k}\right) u, v\right\rangle \leq C\|u\|_{V}\|v\|_{V}
$$

then

$$
\left\|-\Delta-\left(\frac{2}{k^{2}}+\frac{\eta}{k}\right)\right\|_{*} \leq C
$$


second if $u_{j} \longrightarrow u$ weakly in $K$, as $j \longrightarrow+\infty$, then

$\lim \left(-\Delta-\left(\frac{2}{k^{2}}+\frac{\eta}{k}\right)\right)\left(u_{j}\right)=\left(-\Delta-\left(\frac{2}{k^{2}}+\frac{\eta}{k}\right)\right)(u)$,

for any $-\Delta-\left(\frac{2}{k^{2}}+\frac{\eta}{k}\right) \in V^{\prime}$ since

$$
\begin{array}{r}
\lim \sup \left\langle\left(-\Delta-\left(\frac{2}{k^{2}}+\frac{\eta}{k}\right)\right)\left(u_{j}\right), u_{j}-v\right\rangle= \\
\liminf \left\langle\left(-\Delta-\left(\frac{2}{k^{2}}+\frac{\eta}{k}\right)\right)\left(u_{j}\right), u_{j}-v\right\rangle= \\
\left\langle\left(-\Delta-\left(\frac{2}{k^{2}}+\frac{\eta}{k}\right)\right)(u), u-v\right\rangle
\end{array}
$$

$\forall v \in K$, then

$$
\begin{array}{r}
\liminf \left\langle\left(-\Delta-\left(\frac{2}{k^{2}}+\frac{\eta}{k}\right)\right)\left(u_{j}\right), u_{j}-v\right\rangle \geq \\
\left\langle\left(-\Delta-\left(\frac{2}{k^{2}}+\frac{\eta}{k}\right)(u), u-v\right\rangle\right.
\end{array}
$$

then by applying the Theorem 3, we deduce the system (90) has a unique solution. We say that the system (90) is a semi-discrete approximation of the inequalitys (84), (85), (86).

\section{Proof of the Existence}

We introduce

$$
u_{k}(t)=u^{n} \text { in }[n k,(n+1) k[, n=0, \cdots, N-1
$$

then we prove the Lemma

Lemma 13. We take $k \longrightarrow 0$, we have

$$
\begin{aligned}
& u_{k} \text { remain in a bounded set of } L^{\infty}(0, T ; V), \\
& u_{k}^{\prime} \text { remain in a bounded set of } L^{\infty}(0, T ; H) .
\end{aligned}
$$

Proof. We refer to ([13]), ([3], P. 222), ([5]) for the proof of Lemma 13.

we want to solve the initial-value problem such that $\eta \in \mathbb{R}$

$$
\begin{array}{r}
\left.\frac{\partial^{2} u}{\partial t^{2}}+\eta \frac{\partial u}{\partial t}-\Delta u=f, \text { on }\right] 0, T[ \\
u(0)=u_{0}, \frac{\partial u}{\partial t}(0)=u_{1} .
\end{array}
$$

Where $f \in L^{2}(0, T ; H), u_{0} \in K, u_{1} \in H$.

Since the space $V$ is a separable space and consider a sequence of linearly 
independent elements of $V, w_{1}, \cdots, w_{k}, \cdots$, which is total in $V$. Using the Faedo- Galerkin method we define for each $k$ an approximate solution $u_{k}$ of $(93)-(94)$

$$
u_{k}(t)=\sum_{i=1}^{k} g_{i k}(t) w_{i},
$$

then

$$
\begin{aligned}
\frac{\partial^{2}}{\partial t^{2}}\left(u_{k}, w_{j}\right)+\eta \frac{\partial}{\partial t}\left(u_{k}, w_{j}\right) & +a\left(u_{k}, w_{j}\right)=\left(f, w_{j}\right), \\
j=1, \cdots, k, & \\
u_{k}(0) & =u_{0 k}, \\
u_{k}^{\prime}(0) & =u_{1 k} .
\end{aligned}
$$

Where $u_{0 k}$ is the projection in $V$ of $u_{0}$ on the space spanned by $w_{1}, \cdots, w_{k}$, and $u_{1 k}$ is the projection in $V$ of $u_{1}$ on the space spanned by $w_{1}, \cdots, w_{k}$. Equations (95) - (96) - (97) - (98) are equivalent to a linear initial-value problem for an ordinary $k$-dimensional differential equation. They possess a unique solution defined for all time and in particular on $[0, T]$, the function $u_{k}$ is in $\mathscr{C}([0, T] ; V)$ and $u_{k}^{\prime} \in \mathscr{C}([0, T] ; V)$, $u_{k}^{\prime \prime}$ is in $L^{2}(0, T ; V)$.

A priori estimates are obtained by multiplying (96) by $g_{j k}^{\prime}$ and summing these relations for $j=1, \cdots, k$. We obtain

$$
\begin{gathered}
\left(u_{k}^{\prime \prime}, u_{k}^{\prime}\right)+\eta\left(u_{k}^{\prime}, u_{k}^{\prime}\right)+a\left(u_{k}, u_{k}^{\prime}\right)=\left(f, u_{k}^{\prime}\right) . \\
\frac{d}{d t}\left[\left\|u_{k}^{\prime}\right\|^{2}+a\left(u_{k}, u_{k}\right)\right]+2 \eta\left\|u_{k}^{\prime}\right\|^{2}=2\left(f, u_{k}^{\prime}\right) \leq\|f\|^{2}+\left\|u_{k}^{\prime}\right\|^{2} .
\end{gathered}
$$

We use the Lemma 1, we obtain

$$
\begin{aligned}
& u_{k} \text { remain in a bounded set of } L^{\infty}(0, T ; V), \\
& u_{k}^{\prime} \text { remain in a bounded set of } L^{\infty}(0, T ; H) .
\end{aligned}
$$

Then we have (92).

Thus there exists a subsequence, remain denoted $u_{k}$, and $u$, such that

$$
u \in L^{\infty}(0, T ; V), u^{\prime} \in L^{\infty}(0, T ; H),
$$

as $k \longrightarrow+\infty$,

$$
u_{k} \longrightarrow u \text { in } L^{\infty}(0, T ; V) \text { weak-star, }
$$




$$
u_{k}^{\prime} \longrightarrow u^{\prime} \text { in } L^{\infty}(0, T ; H) \text { weak-star } .
$$

Then passing to the limit in $(95)-(96)-(97)-(98)$ we see that $u$ is a solution of (93) - (94) which satisfies (101).

To conclude the proof of existence it remains to show the continuity properties $u \in \mathscr{C}([0, T] ; V), u^{\prime} \in \mathscr{C}([0, T] ; H)$.

We give the following Lemma

Lemma 14. Let $X$ and $Y$ be two Banach spaces such that

$$
X \subset Y
$$

with a continuous injection.

If a function $\varphi$ belongings to $L^{\infty}(0, T ; X)$ and is weakly continuous with values in $Y$, then $\varphi$ is weakly continuous with values in $X$.

The proof of the Lemma 14 is in ([12]), and in ([14], Lemma 1.4, Chapter III).

It follows from the Lemma 14 and (101) that $u$ is weakly continuous from $[0, T]$ in $V$. Similarly, we infer from (93) that

$$
u^{\prime \prime}=f-\eta u^{\prime}+\Delta u
$$

and $u^{\prime \prime} \in L^{2}\left(0, T ; V^{\prime}\right)$, since $f \in L^{2}(0, T ; H), u^{\prime} \in L^{\infty}(0, T ; H)$, $u \in L^{\infty}(0, T ; V)$ which implies $-\Delta u \in L^{\infty}\left(0, T ; V^{\prime}\right)$.

We give the following Lemma

Lemma 15. Let $X$ be a given Banach space with dual $X^{\prime}$ and let $u$ and $g$ be two functions belonging to $L^{1}(0, T ; X)$. Then the following three conditions are equivalent

(i) $u$ is almost everywhere equal to a primitive function $g$, there exists $\xi \in X$ such that

$$
u(t)=\xi+\int_{0}^{t} g(s) d s, \text { for almost everywhere } t \in[0, T] .
$$

(ii) For every test function $\varphi \in \mathscr{D}(] 0, T[)$,

$$
\int_{0}^{T} u(t) \varphi^{\prime}(t) d t=-\int_{0}^{T} g(t) \varphi(t) d t
$$

(iii) For each $\xi \in X^{\prime}$,

$$
\frac{d}{d t}\langle u, \xi\rangle=\langle g, \xi\rangle
$$


in the scalar distribution sense on $] 0, T[$.

If $(i)-(i i)-(i i i)$ are satisfied we say that $g$ is the $X$-valued distribution derivative of $u$, and $u$ is almost everywhere equal to a continuous function from $[0, T]$ into $X$.

The proof of the Lemma 15 is in ([14], Lemma 1.1, Chapter III).

From Lemma 15, then shows that $u$ is continuous from $[0, T]$ in $V^{\prime}$, Lemma 14 and (101) imply that $u^{\prime}$ is weakly continuous from $[0, T]$ in $H$.

We give the following Lemma

Lemma 16. We assume that $w$ is such that

$$
w \in L^{2}(0, T ; V), w^{\prime} \in L^{2}(0, T ; H),
$$

and

$$
w^{\prime \prime}-\Delta w \in L^{2}(0, T ; H) .
$$

Then, after modification on a set of measure zero, $u$ is continuous from $[0, T]$ into $V, u^{\prime}$ is continuous from $[0, T]$ into $H$ and, in the sense of distributions on ] $0, T[$,

$$
\left(w^{\prime \prime}-\Delta w, w^{\prime}\right)=\frac{1}{2} \frac{d}{d t}\left\{\left\|w^{\prime}\right\|^{2}+a(w, w)\right\} .
$$

The proof of the Lemma 16 is in ([13], P.79).

We deduce from Lemma 16 that $u$ satisfies an equation similar to (100), namely

$$
\frac{d}{d t}\left[\left\|u^{\prime}\right\|^{2}+a(u, u)\right]+2 \eta\left\|u^{\prime}\right\|^{2}=2\left(f, u^{\prime}\right)
$$

This shows that the function

$$
t \longmapsto\left\|u^{\prime}(t)\right\|^{2}+a(u(t), u(t))
$$

is continuous on $[0, T]$. In conjunction with the above properties of weak continuity, we conclude that $u \in \mathscr{C}([0, T] ; V)$ and $u^{\prime} \in \mathscr{C}([0, T] ; H)$, then we have $(82)$

$$
u \in \mathscr{C}([0, T] ; V) \bigcap \mathscr{C}^{1}([0, T] ; H)
$$

For show that (83) we have if the set $K$ is a closed and convex of functions $v \in \mathscr{C}([0, T] ; V)$ such that $v(t) \in K$ almost everywhere, then we have $u_{k} \in K$ for any $k$ and since $K$ is weakly closed in $\mathscr{C}([0, T] ; V)$, we have $u \in K$. And from (82) we have $u^{\prime}(t) \in H$.

For show that (84), (85), (86), we consider the function $v$ satisfies

$$
v \in \mathscr{C}^{2}([0, T] ; V), v(t) \in K, v^{\prime}(t) \in H, \text { for any } t \in[0, T]
$$


we put

$$
\begin{gathered}
v^{n}=v(n k), n=0, \cdots, N-1, \\
\left.v_{k}=\ll \text { step function defined by } v_{k}(t)=v^{n} \text { in }\right] n k,(n+1) k[\gg \\
\tilde{v}_{k}=\ll \text { piecewise linear function, continuous in }[0, T]
\end{gathered}
$$

such that

$$
\tilde{v}_{k}(n k)=v^{n-1}, n=1,2, \cdots \text { and } \tilde{v}_{k}(0)=v^{0} \gg .
$$

We note that

$$
\begin{array}{r}
\int_{0}^{T}\left(\frac{d^{2} \tilde{v}_{k}}{d t^{2}}+\eta \frac{d \tilde{v}_{k}}{d t}, v_{k}-u_{k}\right) d t= \\
\Sigma_{n=0}^{N-1} \int_{n k}^{(n+1) k}\left(\frac{d^{2} \tilde{v}_{k}}{d t^{2}}+\eta \frac{d \tilde{v}_{k}}{d t}, v_{k}-u_{k}\right) d t= \\
\Sigma_{n=1}^{N-1}\left(\left(v^{n+1}-2 v^{n}+v^{n-1}\right)+\eta\left(v^{n+1}-v^{n}\right), v^{n}-u^{n}\right),
\end{array}
$$

and that

$$
\int_{0}^{T}\left\langle-\Delta u_{k}, v_{k}-u_{k}\right\rangle d t=k^{2} \Sigma_{n=0}^{N-1}\left\langle-\Delta u^{n}, v^{n}-u^{n}\right\rangle .
$$

We define

$$
f_{k}=f^{n} \text { in }[n k,(n+1) k[, \quad n=0, \cdots, N-1,
$$

we have

$$
\int_{0}^{T}\left\langle f_{k}, v_{k}-u_{k}\right\rangle d t=k^{2} \Sigma_{n=0}^{N-1}\left\langle f^{n}, v^{n}-u^{n}\right\rangle .
$$

We take $v=v^{n}$ in the system (90), and we multiply by $k^{2}$ we obtain that

$$
\begin{array}{r}
\left(\left(u^{n+1}-2 u^{n}+u^{n-1}\right)+\eta k\left(u^{n+1}-u^{n}\right), v^{n}-u^{n}\right)+ \\
k^{2}\left\langle-\Delta u^{n}-f^{n}, v^{n}-u^{n}\right\rangle \geq 0,
\end{array}
$$

then

$$
\begin{array}{r}
\left(\left(v^{n+1}-2 v^{n}+v^{n-1}\right)+\eta k\left(v^{n+1}-v^{n}\right), v^{n}-u^{n}\right)+ \\
k^{2}\left\langle-\Delta u^{n}-f^{n}, v^{n}-u^{n}\right\rangle= \\
\left(\left(u^{n+1}-2 u^{n}+u^{n-1}\right)+\eta k\left(u^{n+1}-u^{n}\right), v^{n}-u^{n}\right)+ \\
k^{2}\left\langle-\Delta u^{n}-f^{n}, v^{n}-u^{n}\right\rangle+ \\
\frac{1+\eta k}{2}\left[\left\|v^{n+1}-u^{n+1}\right\|^{2}-\left\|v^{n}-u^{n}\right\|^{2}-\right. \\
\left.\left\|v^{n+1}-u^{n+1}-\left(v^{n}-u^{n}\right)\right\|^{2}\right]+
\end{array}
$$




$$
\begin{array}{r}
\frac{1}{2}\left[\left\|v^{n-1}-u^{n-1}\right\|^{2}-\left\|v^{n}-u^{n}\right\|^{2}-\right. \\
\left.\left\|v^{n-1}-u^{n-1}-\left(v^{n}-u^{n}\right)\right\|^{2}\right]
\end{array}
$$

From the properties of the norm in $L^{2}(\Omega)$, we have the inequality

$$
\left\|v^{n+1}-u^{n+1}-\left(v^{n}-u^{n}\right)\right\|^{2} \geq\left\|v^{n+1}-u^{n+1}\right\|^{2}-\left\|v^{n}-u^{n}\right\|^{2}
$$

if we have the case $1 .:\left\|v^{n+1}-u^{n+1}\right\|^{2}-\left\|v^{n}-u^{n}\right\|^{2}<0$,

and $\frac{1+\eta k}{2}<0$

by using the case $\mathbf{1}$., on the member

$$
\frac{1+\eta k}{2}\left[\left\|v^{n+1}-u^{n+1}\right\|^{2}-\left\|v^{n}-u^{n}\right\|^{2}-\left\|v^{n+1}-u^{n+1}-\left(v^{n}-u^{n}\right)\right\|^{2}\right]
$$

of the equality (115) and using the inequality (116), we obtain the inequality

$$
\begin{array}{r}
\frac{1+\eta k}{2}\left[\left\|v^{n+1}-u^{n+1}\right\|^{2}-\left\|v^{n}-u^{n}\right\|^{2}-\right. \\
\left.\left\|v^{n+1}-u^{n+1}-\left(v^{n}-u^{n}\right)\right\|^{2}\right] \geq \\
\left\|v^{n+1}-u^{n+1}\right\|^{2}-\left\|v^{n}-u^{n}\right\|^{2}
\end{array}
$$

if we have the case 2 :

$\left\|v^{n-1}-u^{n-1}\right\|^{2}-\left\|v^{n}-u^{n}\right\|^{2}<0$ or $\left\|v^{n-1}-u^{n-1}\right\|^{2}-\left\|v^{n}-u^{n}\right\|^{2}>0$

by using the case 2 ., on the member

$$
\frac{1}{2}\left[\left\|v^{n-1}-u^{n-1}\right\|^{2}-\left\|v^{n}-u^{n}\right\|^{2}-\left\|v^{n-1}-u^{n-1}-\left(v^{n}-u^{n}\right)\right\|^{2}\right]
$$

of the equality (115) and using the inequality (116), we obtain the inequality

$$
\begin{gathered}
\frac{1}{2}\left[\left\|v^{n-1}-u^{n-1}\right\|^{2}-\left\|v^{n}-u^{n}\right\|^{2}-\right. \\
\left.\left\|v^{n-1}-u^{n-1}-\left(v^{n}-u^{n}\right)\right\|^{2}\right] \geq \\
-\left\|v^{n-1}-u^{n-1}-\left(v^{n}-u^{n}\right)\right\|^{2}
\end{gathered}
$$

by using the inequalitys $(114)-(117)-(118)$, on the equality (115) we obtain

$$
\begin{array}{r}
\left(\left(v^{n+1}-2 v^{n}+v^{n-1}\right)+\eta k\left(v^{n+1}-v^{n}\right), v^{n}-u^{n}\right)+ \\
k^{2}\left\langle-\Delta u^{n}-f^{n}, v^{n}-u^{n}\right\rangle \geq \\
\left\|v^{n+1}-u^{n+1}\right\|^{2}-\left\|v^{n}-u^{n}\right\|^{2}- \\
\left\|v^{n-1}-u^{n-1}-\left(v^{n}-u^{n}\right)\right\|^{2}
\end{array}
$$


summing to $n$, we deduce

$$
\begin{array}{r}
\Sigma_{n=1}^{N-1}\left[\left(\left(v^{n+1}-2 v^{n}+v^{n-1}\right)+\eta k\left(v^{n+1}-v^{n}\right), v^{n}-u^{n}\right)+\right. \\
\left.k^{2}\left\langle-\Delta u^{n}-f^{n}, v^{n}-u^{n}\right\rangle\right] \geq \\
\left\|v^{N}-u^{N}\right\|^{2}-\left\|v^{1}-u^{1}\right\|^{2}- \\
\Sigma_{n=1}^{N-1}\left\|v^{n-1}-u^{n-1}-\left(v^{n}-u^{n}\right)\right\|^{2} \geq \\
-\left\|v^{1}-u^{1}\right\|^{2}- \\
\Sigma_{n=1}^{N-1}\left\|v^{n-1}-u^{n-1}-\left(v^{n}-u^{n}\right)\right\|^{2}
\end{array}
$$

with $(110)-(111)-(113)$, we conclude of (120) the inequality

$$
\begin{array}{r}
\int_{0}^{T}\left(\frac{d^{2} \tilde{v}_{k}}{d t^{2}}+\eta \frac{d \tilde{v}_{k}}{d t}, v_{k}-u_{k}\right) d t+ \\
\int_{0}^{T}\left\langle-\Delta u_{k}-f_{k}, v_{k}-u_{k}\right\rangle d t-k^{2}\left\langle-\Delta u^{0}, v^{0}-u^{0}\right\rangle+ \\
k^{2}\left\langle f^{0}, v^{0}-u^{0}\right\rangle+\left\|v^{1}-u^{1}\right\|^{2}+ \\
\Sigma_{n=1}^{N-1}\left\|v^{n-1}-u^{n-1}-\left(v^{n}-u^{n}\right)\right\|^{2} \geq 0
\end{array}
$$

as $k \longrightarrow 0, \frac{d^{2} \tilde{v}_{k}}{d t^{2}} \longrightarrow v^{\prime \prime}$ strongly in $L^{2}\left(0, T ; V^{\prime}\right)$, and $\frac{d \tilde{v}_{k}}{d t} \longrightarrow v^{\prime}$ strongly in $\mathscr{C}([0, T] ; H), v_{k} \longrightarrow v$ strongly in $\mathscr{C}([0, T] ; V)$,

$f_{k} \longrightarrow f$ in $L^{2}(0, T ; H)$ and since $-\Delta$ pseudo-monotone, we have as $k \longrightarrow 0$

$$
\liminf \int_{0}^{T}\left\langle-\Delta u_{k}, u_{k}\right\rangle d t \geq \int_{0}^{T}\langle-\Delta u, u\rangle d t
$$

and since $k^{2} f^{0} \longrightarrow 0$ in $H$ as $k \longrightarrow 0$, we conclude of (121) that

$$
\begin{array}{r}
\int_{0}^{T}\left[\left(v^{\prime \prime}+\eta v^{\prime}, v-u\right)+\langle-\Delta u-f, v-u\rangle\right] d t+\left\|v^{1}-u^{1}\right\|^{2}+ \\
\sum_{n=1}^{N-1}\left\|v^{n-1}-u^{n-1}-\left(v^{n}-u^{n}\right)\right\|^{2} \geq 0
\end{array}
$$

for any $v$ satisfies (109).

if $v$ is given as in the inequality (84), there exists $v_{j}$ satisfies the conditions (109) and such that $v_{j} \longrightarrow v$ weakly in $\mathscr{C}([0, T] ; V), v_{j}^{\prime} \longrightarrow v^{\prime}$ weak-star in $\mathscr{C}([0, T] ; H), v_{j}^{\prime \prime} \longrightarrow v^{\prime \prime}$ in $L^{2}\left(0, T ; V^{\prime}\right)$.

We take $v=v_{j}$ in (122) and pass to the limit, we conclude that (84).

If we have the case 3. :

$\left\|v^{n+1}-u^{n+1}\right\|^{2}-\left\|v^{n}-u^{n}\right\|^{2}>0$ and $\frac{1+\eta k}{2}<0$

by using the case 3 ., on the member

$$
\frac{1+\eta k}{2}\left[\left\|v^{n+1}-u^{n+1}\right\|^{2}-\left\|v^{n}-u^{n}\right\|^{2}-\left\|v^{n+1}-u^{n+1}-\left(v^{n}-u^{n}\right)\right\|^{2}\right]
$$


of the equality (115) and using the inequality (116), we obtain the inequality

$$
\begin{array}{r}
\frac{1+\eta k}{2}\left[\left\|v^{n+1}-u^{n+1}\right\|^{2}-\left\|v^{n}-u^{n}\right\|^{2}-\right. \\
\left.\left\|v^{n+1}-u^{n+1}-\left(v^{n}-u^{n}\right)\right\|^{2}\right] \geq \\
-\left\|v^{n+1}-u^{n+1}-\left(v^{n}-u^{n}\right)\right\|^{2}
\end{array}
$$

by using the inequalitys (114) - (123) - (118) on the equality (115) we obtain

$$
\begin{array}{r}
\left(\left(v^{n+1}-2 v^{n}+v^{n-1}\right)+\eta k\left(v^{n+1}-v^{n}\right), v^{n}-u^{n}\right)+ \\
k^{2}\left\langle-\Delta u^{n}-f^{n}, v^{n}-u^{n}\right\rangle \geq \\
-\left\|v^{n+1}-u^{n+1}-\left(v^{n}-u^{n}\right)\right\|^{2}-\left\|v^{n-1}-u^{n-1}-\left(v^{n}-u^{n}\right)\right\|^{2}
\end{array}
$$

summing to $n$, we deduce

$$
\begin{array}{r}
\Sigma_{n=1}^{N-1}\left[\left(\left(v^{n+1}-2 v^{n}+v^{n-1}\right)+\eta k\left(v^{n+1}-v^{n}\right), v^{n}-u^{n}\right)+\right. \\
\left.k^{2}\left\langle-\Delta u^{n}-f^{n}, v^{n}-u^{n}\right\rangle\right] \geq \\
-\Sigma_{n=1}^{N-1}\left\|v^{n+1}-u^{n+1}-\left(v^{n}-u^{n}\right)\right\|^{2}- \\
\Sigma_{n=1}^{N-1}\left\|v^{n-1}-u^{n-1}-\left(v^{n}-u^{n}\right)\right\|^{2}
\end{array}
$$

with $(110)-(111)-(113)$, we conclude of (125) the inequality

$$
\begin{array}{r}
\int_{0}^{T}\left(\frac{d^{2} \tilde{v}_{k}}{d t^{2}}+\eta \frac{d \tilde{v}_{k}}{d t}, v_{k}-u_{k}\right) d t+ \\
\int_{0}^{T}\left\langle-\Delta u_{k}-f_{k}, v_{k}-u_{k}\right\rangle d t- \\
k^{2}\left\langle-\Delta u^{0}, v^{0}-u^{0}\right\rangle+k^{2}\left\langle f^{0}, v^{0}-u^{0}\right\rangle+ \\
\sum_{n=1}^{N-1}\left\|v^{n+1}-u^{n+1}-\left(v^{n}-u^{n}\right)\right\|^{2}+ \\
\Sigma_{n=1}^{N-1}\left\|v^{n-1}-u^{n-1}-\left(v^{n}-u^{n}\right)\right\|^{2} \geq 0
\end{array}
$$

as $k \longrightarrow 0, \frac{d^{2} \tilde{v}_{k}}{d t^{2}} \longrightarrow v^{\prime \prime}$ strongly in $L^{2}\left(0, T ; V^{\prime}\right)$, and $\frac{d \tilde{v}_{k}}{d t} \longrightarrow v^{\prime}$ strongly in $\mathscr{C}([0, T] ; H), v_{k} \longrightarrow v$ strongly in $\mathscr{C}([0, T] ; V)$, $f_{k} \longrightarrow f$ in $L^{2}(0, T, H)$ and since $-\Delta$ pseudo-monotone, we have as $k \longrightarrow 0$

$$
\liminf \int_{0}^{T}\left\langle-\Delta u_{k}, u_{k}\right\rangle d t \geq \int_{0}^{T}\langle-\Delta u, u\rangle d t,
$$

and since $k^{2} f^{0} \longrightarrow 0$ in $H$ as $k \longrightarrow 0$, we conclude of (126) that

$$
\int_{0}^{T}\left[\left(v^{\prime \prime}+\eta v^{\prime}, v-u\right)+\langle-\Delta u-f, v-u\rangle\right] d t+
$$




$$
\begin{gathered}
\sum_{n=1}^{N-1}\left\|v^{n+1}-u^{n+1}-\left(v^{n}-u^{n}\right)\right\|^{2}+ \\
\sum_{n=1}^{N-1}\left\|v^{n-1}-u^{n-1}-\left(v^{n}-u^{n}\right)\right\|^{2} \geq 0
\end{gathered}
$$

for any v satisfies (109).

If $v$ is given as in the inequality (85) there exists $v_{j}$ satisfies the conditions (109) and such that $v_{j} \longrightarrow v$ weakly in $\mathscr{C}([0, T] ; V), v_{j}^{\prime} \longrightarrow v^{\prime}$ weak-star in $\mathscr{C}([0, T] ; H), v_{j}^{\prime \prime} \longrightarrow v^{\prime \prime}$ in $L^{2}\left(0, T ; V^{\prime}\right)$.

We take $v=v_{j}$ in (127) and pass to the limit, we conclude that (85).

If we have the case 4. :

$\left\|v^{n+1}-u^{n+1}\right\|^{2}-\left\|v^{n}-u^{n}\right\|^{2}<0$ and $\frac{1+\eta k}{2}>0$

and the case 5.:

$\left\|v^{n+1}-u^{n+1}\right\|^{2}-\left\|v^{n}-u^{n}\right\|^{2}>0$ and $\frac{1+\eta k}{2}>0$

in the cases 4., 5., we use the inequalitys (114) - (118) on the equality (115) we obtain the inequality

$$
\begin{array}{r}
\left(\left(v^{n+1}-2 v^{n}+v^{n-1}\right)+\eta k\left(v^{n+1}-v^{n}\right), v^{n}-u^{n}\right)+ \\
k^{2}\left\langle-\Delta u^{n}-f^{n}, v^{n}-u^{n}\right\rangle \geq \\
\frac{1+\eta k}{2}\left[\left\|v^{n+1}-u^{n+1}\right\|^{2}-\left\|v^{n}-u^{n}\right\|^{2}-\right. \\
\left.\left\|v^{n+1}-u^{n+1}-\left(v^{n}-u^{n}\right)\right\|^{2}\right]-\left\|v^{n-1}-u^{n-1}-\left(v^{n}-u^{n}\right)\right\|^{2}
\end{array}
$$

summing to $n$, we deduce

$$
\begin{array}{r}
\Sigma_{n=1}^{N-1}\left[\left(\left(v^{n+1}-2 v^{n}+v^{n-1}\right)+\eta k\left(v^{n+1}-v^{n}\right), v^{n}-u^{n}\right)+\right. \\
\left.k^{2}\left\langle-\Delta u^{n}-f^{n}, v^{n}-u^{n}\right\rangle\right] \geq \\
\frac{1+\eta k}{2}\left[\left\|v^{N}-u^{N}\right\|^{2}-\left\|v^{1}-u^{1}\right\|^{2}-\right. \\
\left.\sum_{n=1}^{N-1}\left\|v^{n+1}-u^{n+1}-\left(v^{n}-u^{n}\right)\right\|^{2}\right]- \\
\Sigma_{n=1}^{N-1}\left\|v^{n-1}-u^{n-1}-\left(v^{n}-u^{n}\right)\right\|^{2} \geq \\
\frac{1+\eta k}{2}\left[-\left\|v^{1}-u^{1}\right\|^{2}-\right. \\
\left.\Sigma_{n=1}^{N-1}\left\|v^{n+1}-u^{n+1}-\left(v^{n}-u^{n}\right)\right\|^{2}\right]- \\
\sum_{n=1}^{N-1}\left\|v^{n-1}-u^{n-1}-\left(v^{n}-u^{n}\right)\right\|^{2}
\end{array}
$$

with $(110)-(111)-(113)$, we conclude of (129) the inequality

$$
\begin{array}{r}
\int_{0}^{T}\left(\frac{d^{2} \tilde{v}_{k}}{d t^{2}}+\eta \frac{d \tilde{v}_{k}}{d t}, v_{k}-u_{k}\right) d t+ \\
\int_{0}^{T}\left\langle-\Delta u_{k}-f_{k}, v_{k}-u_{k}\right\rangle d t-k^{2}\left\langle-\Delta u^{0}, v^{0}-u^{0}\right\rangle+
\end{array}
$$




$$
\begin{array}{r}
k^{2}\left\langle f^{0}, v^{0}-u^{0}\right\rangle+\left(\frac{1+\eta k}{2}\right)\left\|v^{1}-u^{1}\right\|^{2}+ \\
\left(\frac{1+\eta k}{2}\right) \Sigma_{n=1}^{N-1}\left\|v^{n+1}-u^{n+1}-\left(v^{n}-u^{n}\right)\right\|^{2}+ \\
\Sigma_{n=1}^{N-1}\left\|v^{n-1}-u^{n-1}-\left(v^{n}-u^{n}\right)\right\|^{2} \geq 0
\end{array}
$$

as $k \longrightarrow 0, \frac{d^{2} \tilde{v}_{k}}{d t^{2}} \longrightarrow v^{\prime \prime}$ strongly in $L^{2}\left(0, T ; V^{\prime}\right)$, and $\frac{d \tilde{v}_{k}}{d t} \longrightarrow v^{\prime}$ strongly in $\mathscr{C}([0, T] ; H), v_{k} \longrightarrow v$ strongly in

$\mathscr{C}([0, T] ; V), f_{k} \longrightarrow f$ in $L^{2}(0, T ; H)$

and since $-\Delta$ pseudo-monotone, we have as $k \longrightarrow 0$

$$
\liminf \int_{0}^{T}\left\langle-\Delta u_{k}, u_{k}\right\rangle d t \geq \int_{0}^{T}\langle-\Delta u, u\rangle d t
$$

and since $k^{2} f^{0} \longrightarrow 0$ in $H$ as $k \longrightarrow 0$, we conclude of (130) that

$$
\begin{array}{r}
\int_{0}^{T}\left[\left(v^{\prime \prime}+\eta v^{\prime}, v-u\right)+\langle-\Delta u-f, v-u\rangle\right] d t+ \\
\left(\frac{1+\eta k}{2}\right)\left\|v^{1}-u^{1}\right\|^{2}+ \\
\left(\frac{1+\eta k}{2}\right) \Sigma_{n=0}^{N-1}\left\|v^{n+1}-u^{n+1}-\left(v^{n}-u^{n}\right)\right\|^{2}+ \\
\Sigma_{n=0}^{N-1}\left\|v^{n-1}-u^{n-1}-\left(v^{n}-u^{n}\right)\right\|^{2} \geq 0
\end{array}
$$

for any $\mathrm{v}$ satisfies (109).

If $v$ is given as in the inequality (86) there exists $v_{j}$ satisfies the conditions (109) and such that $v_{j} \longrightarrow v$ weakly in $\mathscr{C}([0, T] ; V), v_{j}^{\prime} \longrightarrow v^{\prime}$ weak-star in $\mathscr{C}([0, T] ; H), v_{j}^{\prime \prime} \longrightarrow v^{\prime \prime}$ in $L^{2}\left(0, T ; V^{\prime}\right)$.

We take $v=v_{j}$ in (131) and pass to the limit, we conclude that (86).

\section{Proof of the Uniqueness}

\section{Regularity parabolic and variational inequalities hyperbolic}

We approach parabolic equations by elliptic equations the following step is to approach hyperbolic equations by parabolic equations, this is the regularity parabolic method, that allows us to proved the Uniqueness. We apply this method to the evolution inequalities of type hyperbolic or related to operators well-posed of a sense of Petrowski.

\section{Hypotheses}

Let $V, H$ are Hilbert spaces with

$$
V \subset H, V \text { is dense in } H, V \longrightarrow H \text {, continuous. }
$$


From (8), we have $V \subset H \equiv H^{\prime} \subset V^{\prime}$.

Put, for simplicity

$$
\begin{aligned}
L^{2}(0, T ; V)=L^{2}(V), & L^{2}(0, T ; H)=L^{2}(H), \\
L^{2}\left(0, T ; V^{\prime}\right) & =L^{2}\left(V^{\prime}\right) .
\end{aligned}
$$

And put

$$
\begin{aligned}
& \mathscr{V}=L^{2}(0, T ; V \times V)=L^{2}(V) \times L^{2}(V), \\
& \mathscr{H}=L^{2}(0, T ; V \times H)=L^{2}(V) \times L^{2}(H) .
\end{aligned}
$$

Identifying $\mathscr{H}$ to its dual $\mathscr{H}^{\prime}$, we have

$$
\begin{aligned}
\mathscr{V} & \subset \mathscr{H} \equiv \mathscr{H}^{\prime} \subset \mathscr{V}^{\prime}, \\
\text { such that } \mathscr{V}^{\prime} & =L^{2}(V) \times L^{2}\left(V^{\prime}\right) .
\end{aligned}
$$

Operator $A=-\Delta$

We give $A=-\Delta$ with

$$
-\Delta \in \mathscr{L}\left(V, V^{\prime}\right),(-\Delta)^{*}=-\Delta,
$$

and there exists $c, \alpha$ such that

$$
\langle-\Delta v, v\rangle+c\|v\|^{2} \geq \alpha\|v\|_{V}^{2}, c>0, \alpha>0, \forall v \in V
$$

The scalar product on $V$ is

$$
(u, v)_{V}=((-\Delta+c) u, v), u, v \in V .
$$

Operator $-\Delta$ on $L^{2}(V)$

We define the operator $-\Delta$ from $L^{2}(V) \longrightarrow L^{2}\left(V^{\prime}\right)$ is given by

$$
(-\Delta v)(t)=-\Delta(v(t)) \text { almost everywhere. }
$$

Operator $\mathscr{A}$.

We give $k>0$, we define

$$
\mathscr{A}=\left(\begin{array}{cc}
k I & -I \\
-\Delta & k I
\end{array}\right), \mathscr{A} \in \mathscr{L}\left(\mathscr{V}, \mathscr{V}^{\prime}\right)
$$

If $v=\left\{v_{1}, v_{2}\right\} \in \mathscr{V}$, then

$$
\mathscr{A} v=\left\{k v_{1}-v_{2},-\Delta v_{1}+k v_{2}\right\} \in \mathscr{V}^{\prime} .
$$


The scalar product on $V$ is given by (138), the scalar product in $\mathscr{H}$ is given by

$$
(u, v)=\int_{0}^{T}\left[\left(u_{1}, v_{1}\right)_{V}+\left(u_{2}, v_{2}\right)\right] d t
$$

then

$$
\begin{array}{r}
\langle\mathscr{A} v, v\rangle=\int_{0}^{T}\left[\left(k v_{1}-v_{2}, v_{1}\right)_{V}+\left\langle-\Delta v_{1}+k v_{2}, v_{2}\right\rangle\right] d t= \\
\int_{0}^{T}\left[k\left((-\Delta+c) v_{1}, v_{1}\right)-\left((-\Delta+c) v_{2}, v_{1}\right)+\right. \\
\left.\left\langle-\Delta v_{1}, v_{2}\right\rangle+k\left(v_{2}, v_{2}\right)\right] d t \geq \\
\int_{0}^{T}\left[k \alpha\left\|v_{1}\right\|_{V}^{2}+k\left\|v_{2}\right\|^{2}-c\left\|v_{1}\right\|\left\|v_{2}\right\|\right] d t
\end{array}
$$

but

$$
\|v\| \leq d\|v\|_{V}, \forall v \in V, d>0
$$

then we conclude that if

$$
k>\frac{c d}{2 \sqrt{\alpha}}
$$

then

$$
\text { there exists } \alpha_{0}>0 \text { such that }
$$

$$
\langle\mathscr{A} v, v\rangle \geq \alpha_{0}\|v\|_{\mathscr{V}}^{2}, \forall v \in \mathscr{V} \text {. }
$$

The semigroups $G(s)$ and $g(s)$

We give the semigroup $G(s)$ in $L^{2}(V), L^{2}(H)$ and $L^{2}\left(V^{\prime}\right)$.

$G(s)$ is a semigroup of contractions in $L^{2}(H)$.

We denote by $-\Lambda$ is the infinitesimal generator of $G(s)$, and

$D\left(\Lambda ; L^{2}(H)\right)$ is the domain of $\Lambda$ in $L^{2}(H)$. We can associate to $G(s)$ the semigroup $g(s)$ in $\mathscr{V}, \mathscr{H}, \mathscr{V}^{\prime}$, is given by

$$
g(s)=\left(\begin{array}{cc}
G(s) & 0 \\
0 & G(s)
\end{array}\right)
$$

and we denote by $-L$ is the infinitesimal generator of $g(s)$, is given by

$$
L=\left(\begin{array}{cc}
\Lambda & 0 \\
0 & \Lambda
\end{array}\right)
$$

with the domain

$$
D(L ; \mathscr{H})=D\left(\Lambda ; L^{2}(V)\right) \times D\left(\Lambda ; L^{2}(H)\right) .
$$




\section{The convexs $\mathscr{K}_{i}$}

We give the convexs $\mathscr{K}_{i}, i=1,2$ with

$$
\text { the set } \mathscr{K}_{i} \text {, is a convex closed of } L^{2}(V), 0 \in \mathscr{K}_{i}, i=1,2
$$

and we assume

$$
\begin{aligned}
& \text { there exists } \sigma>0 \text { and } w_{0} \in L^{2}(V) \text { such that } \\
& \sigma \mathscr{K}_{2}+w_{0} \subset \mathscr{K}_{1} .
\end{aligned}
$$

Remark 17. In the applications, using the set $\mathscr{K}_{1}=L^{2}(V)$, such that (148) is satisfying for any $\mathscr{K}_{2}$.

\section{Compatibility}

Perform the following hypotheses

$$
\begin{array}{r}
G(s)(-\Delta)(v)=-\Delta G(s) v, \forall s \geq 0, \forall v \in L^{2}(V), \\
G(s) \mathscr{K}_{i} \subset \mathscr{K}_{i}, \forall s \geq 0, i=1,2,
\end{array}
$$

there exists $\rho>0$ such that, $\forall s \geq 0, \forall v \in \mathscr{K}_{i}, i=1,2$,

$$
G(s) v+G^{*}(s) v-G^{*}(s) G(s) v+(\rho-1) v \in \rho \mathscr{K}_{i} .
$$

Such that $G^{*}(s)$ is the adjoint semigroup of $G(s)$.

We give the following Theorem

Theorem 18. We assume that $-\Delta$ is given with $(136)-(137)$ and $\mathscr{A}$ is given by (140) with (142). We assume that (147) - (148) - (149) - (150) - (151) are satisfied. With another

$$
\int_{0}^{T}\left((-\Delta+c) \Lambda v_{1}, v_{1}\right) d t \geq 0, \forall v_{1} \in D\left(\Lambda ; L^{2}(V)\right) .
$$

Let $f \in D\left(\Lambda ; L^{2}(H)\right)$ and put $F=\{0, f\} \in \mathscr{H}$.

Then there exists a unique function $u$ satisfies

$$
\begin{array}{r}
u \in \mathscr{K}_{1} \times \mathscr{K}_{2}, u \in D(L ; \mathscr{K}), \text { and } \\
(L u, v-u)+\langle\mathscr{A} u, v-u\rangle \geq(F, v-u), \forall v \in \mathscr{K} .
\end{array}
$$

The proof of the Theorem is in ([3], P. 349).

From the proof of the Theorem 18 we give the following remarks 
Remark 19. The variational inequality (154) is not of type parabolic because $\mathscr{A}$ is restrict to $\mathscr{V}$ is not coercive on $\mathscr{V}$, but is only coercive on $\mathscr{H}$, this is the situation typical of hyperbolic operator or well-posed of a sense of Petrowski.

Remark 20. Before we give applications expounding (154), since $\mathscr{K}=$ $\mathscr{K}_{1} \times \mathscr{K}_{2}$, then from (154) we have the inequalitys

$$
\begin{array}{r}
u_{1} \in D\left(\Lambda ; L^{2}(V)\right), u_{1} \in \mathscr{K}_{1}, \\
\int_{0}^{T}\left[\left(\Lambda u_{1}, v_{1}-u_{1}\right)_{V}+\left(k u_{1}-u_{2}, v_{1}-u_{1}\right)_{V}\right] d t \geq 0, \\
\forall v_{1} \in \mathscr{K}_{1}, \\
u_{2} \in D\left(\Lambda ; L^{2}(H)\right) \bigcap L^{2}(V), u_{2} \in \mathscr{K}_{2}, \\
\int_{0}^{T}\left[\left(\Lambda u_{2}, v_{2}-u_{2}\right)+\left\langle-\Delta u_{1}+k u_{2}, v_{2}-u_{2}\right\rangle\right] d t \geq \\
\int_{0}^{T}\left(f, v_{2}-u_{2}\right) d t, \forall v_{2} \in \mathscr{K}_{2} .
\end{array}
$$

In particular, $\mathscr{K}_{1}=L^{2}(V)$ then from (155) we deduce the equation

$$
\Lambda u_{1}+k u_{1}-u_{2}=0 \text {. }
$$

Remark 21. Third a priori estimate in the proof of the Theorem 18 is not satisfying if

$$
\mathscr{K}_{2} \text { is bounded in } L^{2}(V)
$$

then (148) is not utility.

Remark 22. Since $\mathscr{A}$ is given by (140), but with $k=0$ we have

$$
\mathscr{A}=\left(\begin{array}{cc}
0 & -I \\
-\Delta & 0
\end{array}\right)
$$

and perform the following hypothese : $\mathscr{K}_{1}=L^{2}(V), \mathscr{K}_{2}$ is bounded in $L^{2}(V)$ and if $u_{j} \in D\left(\Lambda ; L^{2}(V)\right)$ and $(\Lambda+\epsilon) v_{j}$ is remain in bounded of $L^{2}(V)$, then $v_{j}$ is remain in a bounded set of $L^{2}(V)$, for any $j$, as $j \longrightarrow 0$.

In these conditions, the Theorem 18 is also satisfying.

Indeed, by taking

$$
\mathscr{B}=\left(\begin{array}{cc}
I & 0 \\
0 & -\Delta+c
\end{array}\right)
$$


such that

$$
\langle(\mathscr{A}+\epsilon \mathscr{B}) v, v\rangle=\epsilon\langle\mathscr{B} v, v\rangle \geq \epsilon\|v\|_{\mathscr{V}}^{2},
$$

then there exists $u_{\epsilon}$ in $\mathscr{K}_{1} \times \mathscr{K}_{2}$ and $D(L ; \mathscr{V})$ such that there exists

$$
\left(L u_{\epsilon}, v-u_{\epsilon}\right)+\left\langle(\mathscr{A}+\epsilon \mathscr{B}) u_{\epsilon}, v-u_{\epsilon}\right\rangle \geq\left(F, v-u_{\epsilon}\right), \forall v \in \mathscr{K},
$$

then

$$
\left((-\Delta+c)\left(\Lambda u_{\epsilon 1}-u_{\epsilon 2}+\epsilon u_{\epsilon 1}\right), v_{1}-u_{\epsilon 1}\right) \geq 0, \forall v_{1} \in L^{2}(V)
$$

and then

$$
(\Lambda+\epsilon) u_{\epsilon 1}=u_{\epsilon 2} .
$$

But $u_{\epsilon 2} \in \mathscr{K}_{2}$ bounded in $L^{2}(V)$ and then $u_{\epsilon 1}$ is remain in a bounded set of $L^{2}(V)$ and bounded of $D\left(\Lambda, L^{2}(V)\right)$.

Then we have the results of first and third a priori estimate in the proof of the Theorem 18, second a priori estimate is unchanged.

Remark 23. From the proof of Theorem 18 can be replaced The hypotheses (150) - (151) by the following hypotheses respectively,

there exists $\beta \in \mathbb{R}$, such that $\forall s \geq 0, e^{\beta s} G(s) \mathscr{K}_{i} \subset \mathscr{K}_{i}$,

$$
i=1,2,
$$

there exists $\rho>0$, such that, $\forall s \geq 0, \forall v \in \mathscr{K}_{i}, i=1,2$,

$e^{\beta s} G(s) v+e^{-\beta s} G^{*}(s) v=G^{*}(s) G(s) v+(\rho-1) v \in \rho \mathscr{K}_{i}$.

\section{First application}

From the Theorem 18 we prove as application the following Theorem.

Theorem 24. We give the function $f=f(x, t)$, with

$$
f, \frac{\partial f}{\partial t} \in L^{2}(Q), f(x, 0)=0 .
$$

There exists a unique function $u$ satisfies

$$
\begin{array}{r}
u, \frac{\partial u}{\partial x_{i}}, \frac{\partial u}{\partial t}, \frac{\partial^{2} u}{\partial x_{i} \partial t}, \frac{\partial^{2} u}{\partial t^{2}} \in L^{2}(Q), i=1, \cdots, n, \\
\frac{\partial^{2} u}{\partial t^{2}}-\Delta u=f, \text { in } Q
\end{array}
$$




$$
\begin{gathered}
u(x, 0)=0, \frac{\partial u}{\partial t}(x, 0)=0, \text { on } \Omega, \\
\frac{\partial u}{\partial t} \geq 0 \text { on } \Sigma, \frac{\partial u}{\partial n} \geq 0 \text { on } \Sigma, \\
\frac{\partial u}{\partial t} \times \frac{\partial u}{\partial n}=0, \text { on } \Sigma .
\end{gathered}
$$

Such that $\frac{\partial}{\partial n}=$ normal derivative to $\partial \Omega$, directed on the exterior of $\Omega$. From (165), $\Delta u=\frac{\partial^{2} u}{\partial t^{2}}-f \in L^{2}(Q)$, such that $\frac{\partial u}{\partial n}$ has a sense from ([4]).

Proof. We apply the Theorem 18 in the following conditions

$$
\begin{gathered}
V=H^{1}(\Omega), \\
(-\Delta u, v)=\Sigma_{i=1}^{n} \int_{\Omega} \frac{\partial u}{\partial x_{i}} \frac{\partial v}{\partial x_{i}} d x, \\
\mathscr{K}_{1}=L^{2}(V)=L^{2}(0, T ; V), \\
\mathscr{K}_{2}=\left\{v \mid v \in L^{2}(V), v \geq 0, \text { almost everywhere on } \Sigma\right\}, \\
G(s) \varphi(t)=\left\{\begin{array}{r}
\varphi(t-s) \text { if } t \geq s, \\
0, \text { if } t<s .
\end{array}\right.
\end{gathered}
$$

Here the conditions of the Theorem 18 are satisfied, choosing $k>0$. The operator $\Lambda$ is $\Lambda=\frac{\partial}{\partial t}$, with domain the null functions for $t=0$, here by using (157), then we have the existence and uniqueness of the couple $u_{1}, u_{2}$ with

$$
\begin{array}{r}
u_{1} \in D\left(\Lambda ; L^{2}(V)\right), \text { such that, } \\
u_{1} \in L^{2}(V), u_{1}^{\prime} \in L^{2}(V), u_{1}(0)=0, \\
u_{2} \in D\left(\Lambda ; L^{2}(H)\right), u_{2} \in \mathscr{K}_{2}, \\
u_{1}^{\prime}+k u_{1}-u_{2}=0, \\
\int_{0}^{T}\left(u_{2}^{\prime}+(-\Delta) u_{1}+k u_{2}-f, v_{2}-u_{2}\right) d t \geq 0, \\
\forall v_{2} \in \mathscr{K}_{2} .
\end{array}
$$

As $\mathscr{K}_{2}$ is cone of summit the origin, (172) is equivalent to

$$
\int_{0}^{T}\left(u_{2}^{\prime}+(-\Delta) u_{1}+k u_{2}-f, v_{2}\right) d t \geq 0
$$




$$
\begin{array}{r}
\forall v_{2} \in \mathscr{K}_{2}, \text { with } \int_{0}^{T}\left(u_{2}^{\prime}+(-\Delta) u_{1}+k u_{2}-f, v_{2}\right) d t=0, \\
\text { if } v_{2}=u_{2} .
\end{array}
$$

Using the definition of $\mathscr{K}_{2}$, we conclude that

$$
u_{2}^{\prime}-\Delta u_{1}+k u_{2}=f, \text { in } Q
$$

If we multiplying by $v_{2}$ and integration by parts, we deduce of (174) that

$$
\int_{\Sigma} \frac{\partial u_{1}}{\partial n} v_{2} d \Sigma=\int_{0}^{T}\left(u_{2}^{\prime}+(-\Delta) u_{1}+k u_{2}-f, v_{2}\right) d t
$$

and then

$$
\begin{array}{r}
\int_{\Sigma} \frac{\partial u_{1}}{\partial n} v_{2} d \Sigma \geq 0, \forall v_{2} \in \mathscr{K}_{2}, \\
\text { with, } \int_{\Sigma} \frac{\partial u_{1}}{\partial n} v_{2} d \Sigma=0, \text { if } v_{2}=u_{2},
\end{array}
$$

then

$$
\frac{\partial u_{1}}{\partial n} \geq 0, u_{2} \frac{\partial u_{1}}{\partial n}=0
$$

Then put

$$
w_{i}=e^{k t} u_{i}, i=1,2 .
$$

We conclude of (171) and (176) that

$$
\begin{array}{r}
w_{1}^{\prime}-w_{2}=0, \\
w_{2}^{\prime}-\Delta w_{1}=e^{k t} f=f^{*}, \\
w_{2} \geq 0, \text { on } \Sigma, \frac{\partial w_{1}}{\partial n}=0, \text { on } \Sigma, \\
w_{2} \frac{\partial w_{1}}{\partial n}=0, \text { on } \Sigma .
\end{array}
$$

Then $u=w_{1}$ satisfying the conditions of Theorem 24 , with $f$ is replaced by $f^{*}$.

As we have equivalent of the research of $u$, of $\left\{w_{1}, w_{2}\right\}$ and $\left\{u_{1}, u_{2}\right\}$ we have further the uniqueness. 


\section{Second application}

We consider the problem with periodic solutions in $t$ of inequality of type (166)(168). We take that the change $w_{i}=e^{k t} u_{i}, i=1,2$, exterminate the periodicity in $t$ then we will verify this.

We give the following Theorem as application on the Theorem 18 and the Theorem 24

Theorem 25. We give the function $f=f(x, t)$, with

$$
f, \frac{\partial f}{\partial t} \in L^{2}(Q), f(x, 0)=f(x, T), x \in \Omega
$$

Let $k=\eta>0$.

There exists a unique function $u$, satisfies (165) and

$$
\begin{array}{r}
\frac{\partial^{2} u}{\partial t^{2}}+k \frac{\partial u}{\partial t}-\Delta u=f, \text { in } Q, \\
u(x, 0)=u(x, T), \\
\frac{\partial u}{\partial t}(x, 0)=\frac{\partial u}{\partial t}(x, T), x \in \Omega,
\end{array}
$$

and (168).

Proof. Applying the Theorem 18 with the same hypotheses of the Theorem 24 , but the semigroup $G(s)$ is given by

$$
G(s) \varphi(t)=\left\{\begin{array}{r}
\varphi(t-s+T), \text { if } t \leq s \\
\varphi(t-s), \text { if } t \geq s
\end{array}\right.
$$

We obtain the existence and uniqueness of the couple $\left\{u_{1}, u_{2}\right\}$ with

$$
\begin{array}{r}
u_{1} \in D\left(\Lambda ; L^{2}(V)\right), \text { such that } u_{1} \in L^{2}(V), u_{1}^{\prime} \in L^{2}(V), \\
u_{1}(x, 0)=u_{1}(x, T), u_{2} \in D\left(\Lambda ; L^{2}(H)\right), u_{2} \in \mathscr{K}_{2},
\end{array}
$$

and $(171)-(172)$. Then interpret $(172)$ as in the Theorem 24 and that $u=$ $u_{1}$ satisfying the conditions of the Theorem 24, as we have equivalent of the research of $u$, of $\left\{u_{1}, u_{2}\right\}$ we have further the uniqueness.

Then we obtain the Uniqueness in the Theorem 12 when $\eta>0$.

To illustrate our results we consider the following example. 


\subsection{An Example}

We consider the initial - boundary value problem

$$
\left\{\begin{array}{r}
\frac{\partial^{2} u}{\partial t^{2}}+\eta \frac{\partial u}{\partial t}-\frac{\partial^{2} u}{\partial x^{2}}=f(x, t), \\
x \in] 0,1[, t \in] 0, T[ \\
u(0, t)=u(1, t)=0, t \in] 0, T[ \\
\left.u(x, 0)=2 \sin \pi x=u_{0}(x), \quad x \in\right] 0,1[ \\
\left.\frac{\partial u}{\partial t}(x, 0)=-\sin 2 \pi x=u_{1}(x), \quad x \in\right] 0,1[
\end{array}\right.
$$

Where $\eta>0$, and $f(x, t)=-2 \eta \pi\left(\sin \pi x \sin \pi t+\frac{1}{2 \pi} \sin 2 \pi x \cos 2 \pi t\right)$ the unique solution of (183) is given by

$$
u(x, t)=2 \sin \pi x \cos \pi t-\frac{1}{2 \pi} \sin 2 \pi x \sin 2 \pi t
$$

For find the unique numerical solution $v$, the way we derive the finite difference scheme for (183) then to replace the derivatives involved in (183) by finite differences. But for (183) we have to approximate both the space and the time derivatives.

Let $n \geq 1$ be a given integer, the grid spacing in the $x$ - direction is $\triangle x=\frac{1}{n+1}$. The grid points are $x_{j}=j \triangle x$ for $j=0,1, \cdots, n+1$.

The discrete time levels are given by $t_{m}=m \Delta t$ for integers $m \geq 0$, where $\triangle t>0$ is the time step.

The grid function $v$, with $v_{j}^{m}=v\left(x_{j}, t_{m}\right)$, approximates $u$.

Then we define the difference scheme

$$
\begin{array}{r}
\frac{v_{j}^{m+1}-2 v_{j}^{m}+v_{j}^{m-1}}{(\triangle t)^{2}}+\eta \frac{v_{j}^{m+1}-v_{j}^{m}}{\triangle t}- \\
\frac{v_{j+1}^{m}-2 v_{j}^{m}+v_{j-1}^{m}}{(\triangle x)^{2}}=f\left(x_{j}, t_{m}\right)
\end{array}
$$

for $j=1,2, \cdots, n$ and for $m \geq 1$. We require the discrete solution to satisfy the boundary conditions in (183),

$$
v_{0}^{m}=v_{n+1}^{m}=0 \quad \text { for, } m \geq 0 .
$$

If $\left\{v_{j}^{m}\right\}_{j=1}^{n}$ and $\left\{v_{j}^{m-1}\right\}_{j=1}^{n}$ are known, then the solutions $\left\{v_{j=1}^{m+1}\right\}_{j=1}^{n}$ can be computed from (185). We need to know $v$ at the first two time levels. We have

$$
v_{j}^{0}=u_{0}\left(x_{j}\right) \quad j=1,2, \cdots, n .
$$


to obtain approximation $v_{j}^{1}$ for $u(x, \triangle t)$ we use a Taylor expansion with respect to time to obtain

$$
\begin{gathered}
u(x, \triangle t)=u(x, 0)+(\triangle t) \frac{\partial u}{\partial t}(x, 0)+\frac{(\triangle t)^{2}}{2} \frac{\partial^{2} u}{\partial t^{2}}(x, 0)+O\left((\triangle t)^{3}\right) \\
u(x, \triangle t)=u_{0}(x)+(\triangle t) u_{1}(x)+\frac{(\triangle t)^{2}}{2} u_{0}^{\prime \prime}(x)+O\left((\triangle t)^{3}\right) .
\end{gathered}
$$

Since

$$
\frac{\partial^{2} u}{\partial t^{2}}(x, 0)=\frac{\partial^{2} u}{\partial x^{2}}(x, 0)=u_{0}^{\prime \prime}(x) .
$$

Then, we have the following approximation $v_{j}^{1}$ for $u\left(x_{j}, \triangle t\right)$

$$
v_{j}^{1}=v_{j}^{0}+(\triangle t) u_{1}\left(x_{j}\right)+\frac{(\triangle t)^{2}}{2(\triangle x)^{2}}\left(v_{j-1}^{0}-2 v_{j}^{0}-v_{j+1}^{0}\right) .
$$

To write (185) in a more compact form, we let $v^{m} \in \mathbb{R}^{n}$ be the vector $v^{m}=$ $\left(v_{1}^{m}, v_{2}^{m}, \cdots, v_{n}^{m}\right)^{\tau}, \tau=$ transferred, and $A \in \mathbb{R}^{n, n}$ the tridiagonal matrix

$$
A=\frac{1}{(\triangle x)^{2}}\left(\begin{array}{ccccc}
2 & -1 & 0 & \cdots & 0 \\
-1 & 2 & -1 & \ddots & \vdots \\
0 & \ddots & \ddots & \ddots & 0 \\
\vdots & \ddots & -1 & 2 & -1 \\
0 & \cdots & 0 & -1 & 2
\end{array}\right)
$$

then (185) can be written for $m \geq 1$,

$$
\begin{array}{r}
v^{m+1}=\left(\frac{2+\eta \triangle t}{1+\eta \triangle t} I-\frac{(\triangle t)^{2}}{1+\eta \triangle t} A\right) v^{m}- \\
\frac{1}{1+\eta \triangle t} v^{m-1}+f^{m}
\end{array}
$$

where $I \in \mathbb{R}^{n, n}$, is the identity matrix, and $f^{m}=\left(f_{1}^{m}, f_{2}^{m}, \cdots, f_{n}^{m}\right)^{\tau}$ with components given by

$$
\begin{array}{r}
f_{j}^{m}=f\left(x_{j}, t_{m}\right)=-\frac{2 \eta \pi(\triangle t)^{2}}{1+\eta \triangle t}\left(\sin \pi x_{j} \sin \pi t_{m}+\right. \\
\left.\frac{1}{2 \pi} \sin 2 \pi x_{j} \cos 2 \pi t_{m}\right)
\end{array}
$$

for $j=1,2, \cdots, n$ and $m \geq 1$, where the initial approximation $v^{0}$ and $v^{1}$ are determined by (186) and (187). 


\section{Acknowledgments}

This work is partially supported by the grants of CNEPRU Code : B 02420120015 Algeria.

\section{References}

[1] H. Brezis, Analyse fonctionnelle, Paris. Masson. (1983).

[2] J. Dabas and D. Bahuguna, Existence and Uniqueness of Solutions of Strongly Damped Wave Equations with Integral Boundary Conditions, Nonlinear Dynamics and Systems Theory, 11 (1) (2011) 65-82.

[3] J. L. Lions, Quelques méthodes de resolution des problémes aux limites Non- linéaires, Éditions Dunod, Paris, 1969.

[4] J. L. Lions, E. Magenes, Problémes aux limites non homogénes et applications, Vol. 1 et 2, Paris, Dunod, 1968.

[5] J. L. Lions, E. Magenes, problémes aux limites non homogénes, (3 volumes), Dunod, (1968).

[6] L. Nirenberg, Remarks on strongly elliptic differential equations, Comm. Pure Applied Math., 8(1955), 648-674.

[7] Hisashi Nishiyama, Polynomial decay for Damped wave Equations on partially Rectangular Domains, Math. Res. Lett. 16 (2009), no.5, 881-894.

[8] L. Schwartz, Théorie des distributions, I,II. Hermann, paris, 1950-1951 (2e édition 1957).

[9] L. Schwartz, Distributions á valeurs vectorielles, I,II Annales Institut Fourier, 7 (1957), 1-141; 8 (1958), 1-209.

[10] R. E. Showalter, Hilbert space Methods for partial Differential equations, Austin, Texas Jaunuary, 1977.

[11] S. I. Sobolev, Applications de l'analyse fonctionnelle aux équations de la physique mathématique, Léningrad, 1950.

[12] W. A. Strauss, On the continuity of functions with values in various Banach spaces, Pacific J. Math., 19, 3 (1966), 543-555.. 
[13] R. Temam, Infinite- Dimensional Dynamical systems in Mechanics and physics, Second edition, Springer, Vol.68, 1977.

[14] R. Temam, Navier-Stokes equations, Theory and numerical analysis, 3rd rev. ed., North-Holland, Amstredam, 1984..

[15] A. Tveito, R. Winther, Introduction to partial Differential equations, a Computation approach, Springer, Oslo, Norway, July 1998.

[16] K. Yosida, Functional Analysis, Grund leheren B. 123, Springer (1965).

[17] Yuncheng You, Global Dynamics of Nonlinear Wave Equations With Cubic Non-Monotone Damping, Dynamics of PDE, Vol. 1, No.1, 65-86, 2004. 